\title{
Modeling two-stage bunch compression with wakefields: Macroscopic properties and microbunching instability
}

\author{
R. A. Bosch and K. J. Kleman \\ Synchrotron Radiation Center, University of Wisconsin-Madison, 3731 Schneider Drive, Stoughton, Wisconsin 53589, USA
}

J. Wu

Stanford Linear Accelerator Center, Stanford University, Stanford, California 94309, USA

(Received 21 December 2007; published 23 September 2008)

\begin{abstract}
In a two-stage compression and acceleration system, where each stage compresses a chirped bunch in a magnetic chicane, wakefields affect high-current bunches. The longitudinal wakes affect the macroscopic energy and current profiles of the compressed bunch and cause microbunching at short wavelengths. For macroscopic wavelengths, impedance formulas and tracking simulations show that the wakefields can be dominated by the resistive impedance of coherent edge radiation. For this case, we calculate the minimum initial bunch length that can be compressed without producing an upright tail in phase space and associated current spike. Formulas are also obtained for the jitter in the bunch arrival time downstream of the compressors that results from the bunch-to-bunch variation of current, energy, and chirp. Microbunching may occur at short wavelengths where the longitudinal space-charge wakes dominate or at longer wavelengths dominated by edge radiation. We model this range of wavelengths with frequency-dependent impedance before and after each stage of compression. The growth of current and energy modulations is described by analytic gain formulas that agree with simulations.
\end{abstract}

DOI: 10.1103/PhysRevSTAB.11.090702

PACS numbers: 29.27.Bd, 41.60.Cr

\section{INTRODUCTION}

A two-stage compression and acceleration system, where each stage under compresses a chirped bunch in a magnetic chicane, may be utilized in a free-electron laser (FEL) driver [1]. For a high-current bunch, longitudinal wakefields affect the macroscopic current profile of the compressed bunch and/or cause microbunching [2-10]. The bunch is affected by coherent-radiation wakes, which are approximated by steady-state coherent synchrotron radiation (CSR) within the chicane magnets [11] and coherent edge radiation (CER) [12-15] downstream of the magnets, in addition to the linac geometric and longitudinal-space-charge (LSC) wakes. When the compressions are performed at sufficiently high beam energy, the longitudinal particle distributions are nearly frozen after each chicane. For this case, we model the effect of longitudinal wakes with effective impedances before and after each chicane.

We apply our model to a preliminary two-stage bunch compressor design for the Wisconsin Free Electron Laser (WiFEL) [16], in which the electron-gun current of $50 \mathrm{~A}$ is increased by a factor of 20 to $1 \mathrm{kA}$. At macroscopic wavelengths of $\sim 2 \pi$ times the bunch length, the calculated impedance is dominated by CER downstream of the chicane magnets. A drift length of several meters contributes a resistive CER impedance of $\sim Z_{0}$, where $Z_{0}=120 \pi \Omega$ is the impedance of free space [14]. Tracking with the code ELEGANT [17] confirms that the macroscopic effects of coherent-radiation wakes are approximated by resistive impedance. We use this approximation to obtain formulas for the minimum initial bunch length that can be compressed without producing an upright tail in phase space, and for the jitter in the bunch arrival time resulting from the bunch-to-bunch variation in current, energy and chirp.

The microbunching caused by wakes is considered for a bunch with nonzero emittance and energy spread. To model long wavelengths where CER dominates as well as short wavelengths where LSC dominates, we consider frequency-dependent impedances before and after each chicane. For a small current or energy modulation at the bunch-compressor entrance, the current and energy modulations of the compressed bunch are described by analytic formulas that agree with tracking simulations of a sixdimensional phase space.

For the preliminary WiFEL bunch compressor design, we calculate the minimum compressible initial bunch length, the jitter from bunch-to-bunch variations in current, energy, and chirp, and the microbunching growth. With state-of-the-art control of the linac's superconducting cavities, we plan to obtain acceptable performance by compressing a 200-pC bunch with peak current of $50 \mathrm{~A}$ and rms bunch length of $400 \mu \mathrm{m}$ by a factor of 20 , obtaining a peak current of $1 \mathrm{kA}$.

\section{A TWO-STAGE BUNCH COMPRESSOR}

For WiFEL [16], we plan to accelerate a bunch from a 4$\mathrm{MeV}$ electron gun that has normalized horizontal emittance $\left(\beta \gamma \varepsilon_{x}\right)$ and vertical emittance $\left(\beta \gamma \varepsilon_{y}\right)$ of $1 \mu \mathrm{m}-\mathrm{rad}$. Here, 
$\beta$ is the velocity divided by the speed of light, $\gamma$ is the relativistic factor while $\varepsilon_{x}$ and $\varepsilon_{y}$ are the geometric emittances. We first consider radiofrequency (rf) parameters that provide good compression when collective effects are neglected. A schematic is shown in Fig. 1(a), while parameters are given in Table I.

The 4-MeV bunch is accelerated to $251.3 \mathrm{MeV}$ in an injector linac composed of 24 TESLA 1.3-GHz superconducting cavities, with the bunch center at a linac phase $17.8^{\circ}$ before the peak. Passage through ten superconducting 3.9-GHz harmonic cavities approximately linearizes the chirp while decelerating the bunch to the energy $E_{1}$ of $215 \mathrm{MeV}$. The chicane $\mathrm{BC} 1$, whose transfer matrix element $R_{56}^{(1)}$ equals $-87 \mathrm{~mm}$ (in the convention where $R_{56}$ is negative for a chicane), compresses the bunch by the ratio $C_{1}=8$ over a length of $12 \mathrm{~m}$. The bunch is then accelerated to the energy $E_{2}$ of $485 \mathrm{MeV}$ in the L1 linac, which consists of sixteen 1.3-GHz cavities, with the bunch at a phase $9^{\circ}$ after the peak. The chicane $\mathrm{BC} 2$, whose transfer matrix element $R_{56}^{(2)}$ is $-18 \mathrm{~mm}$, compresses the bunch by the ratio $C_{2}=2.5$ over a length of $10 \mathrm{~m}$. The fully compressed bunch is then accelerated and dechirped in the L2 linac, which consists of $1201.3-\mathrm{GHz}$ cavities, with the bunch at a phase $50.6^{\circ}$ after peak. Its final energy $E_{f}$ is $1.7 \mathrm{GeV}$.

In Fig. 1(b), we show lattice functions when this compressor is implemented with FODO focusing in the L1 and $\mathrm{L} 2$ linacs, using $5^{\circ}$ bending magnets in $\mathrm{BC} 1$ and $2.5^{\circ}$
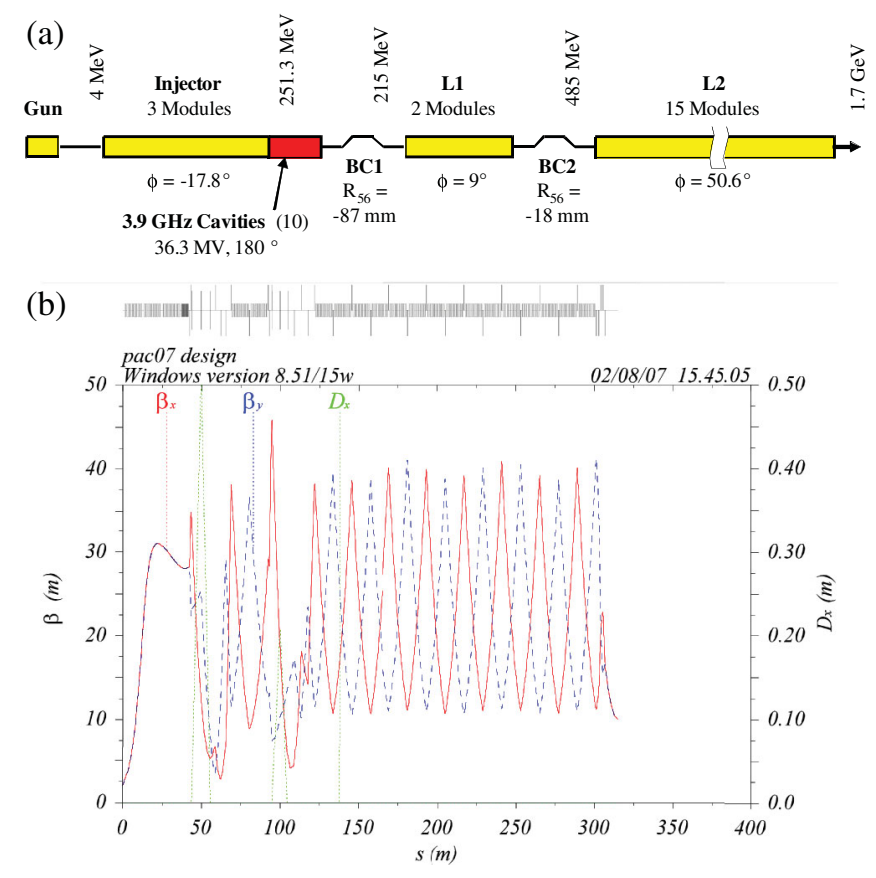

FIG. 1. (Color) (a) Schematic of the WiFEL preliminary bunch compressor design, optimized for zero current or trapezoidal bunches. Each linac module contains eight 1.3-GHz superconducting cavities. (b) Lattice functions plotted by the MAD-withacceleration code.
TABLE I. Parameters describing the chicanes BC1 and BC2 and the compression of 200-pC parabolic bunches.

\begin{tabular}{lcc}
\hline \hline \multicolumn{1}{c}{ Property } & Symbol & Value \\
\hline BC1 energy & $E_{1}$ & $215 \mathrm{MeV}$ \\
BC1 compression ratio & $C_{1}$ & 8 \\
BC1 energy-to-position transfer & $R_{56}^{(1)}$ & $-87 \mathrm{~mm}$ \\
BC2 energy & $E_{2}$ & $485 \mathrm{MeV}$ \\
BC2 compression ratio & $C_{2}$ & 2.5 \\
BC2 energy-to-position transfer & $R_{56}^{(2)}$ & $-18 \mathrm{~mm}$ \\
Initial bunch peak current & $I_{0}$ & $50 \mathrm{~A}$ \\
Peak current after BC1 & $C_{1} I_{0}$ & $400 \mathrm{~A}$ \\
Peak current after BC2 & $C_{1} C_{2} I_{0}$ & $1 \mathrm{kA}$ \\
Initial rms bunch length & $\sigma_{z}$ & $400 \mu \mathrm{m}$ \\
Bunch length after BC1 & $\sigma_{z} / C_{1}$ & $50 \mu \mathrm{m}$ \\
Bunch length after BC2 & $\sigma_{z} / C_{1} C_{2}$ & $20 \mu \mathrm{m}$ \\
Normalized transverse emittance & $\beta \gamma \varepsilon_{x}, \beta \gamma \varepsilon_{y}$ & $1 \mu \mathrm{m}-\mathrm{rad}$ \\
\hline \hline
\end{tabular}

bending magnets in $\mathrm{BC} 2$ whose radius of curvature is $1.83 \mathrm{~m}$. The lattice functions were computed by the MAD-with-acceleration code [18], modified by DESY to include the transverse focusing in a standing wave cavity [19].

Figure 2 shows the longitudinal phase space during compression of a parabolic bunch with rms length $\sigma_{z}$ of $400 \mu \mathrm{m}$, Gaussian energy spread $\sigma_{E}$ of $3 \mathrm{keV}$, and normalized transverse emittances of $1 \mu \mathrm{m}$-rad. In the phasespace plots, the longitudinal coordinate $z$ is zero at the average electron position, with $z>0$ at the tail of the bunch. The tracking of six-dimensional phase space was performed without collective effects by the ELEGANT code [17].

\section{IMPEDANCE}

\section{A. Sources of impedance}

For high bunch currents, the compression is affected by wakes. Consider a current modulation $\Delta I e^{-i \omega t}$, where $\omega$ is the angular frequency, $t$ is the arrival time, $c$ is the speed of light, $k=\omega / c$ is the wave number, and $\lambda$ is the wavelength. The retarding voltage from a longitudinal wake is $Z(\omega) \Delta I e^{-i \omega t}$, where $Z(\omega)$ is the wake's impedance. We consider four sources of longitudinal impedance.

In SI units, the free space longitudinal space charge (LSC) impedance per unit length, expressed as a function of $k$, is given for $k>0$ by [8]

$$
Z_{\mathrm{LSC}}(k)=\frac{i Z_{0}}{\pi k r_{b}^{2}}\left[1-\frac{k r_{b}}{\gamma} K_{1}\left(\frac{k r_{b}}{\gamma}\right)\right]
$$

where $r_{b}$ is the radius of a uniform round electron bunch and $K_{1}$ is a modified Bessel function. To approximate the impedance for Gaussian or parabolic transverse distributions with rms dimensions $\sigma_{x}$ and $\sigma_{y}$, Eq. (1) may be applied with effective radius $r_{b}=0.85\left(\sigma_{x}+\sigma_{y}\right)$ [8]. 

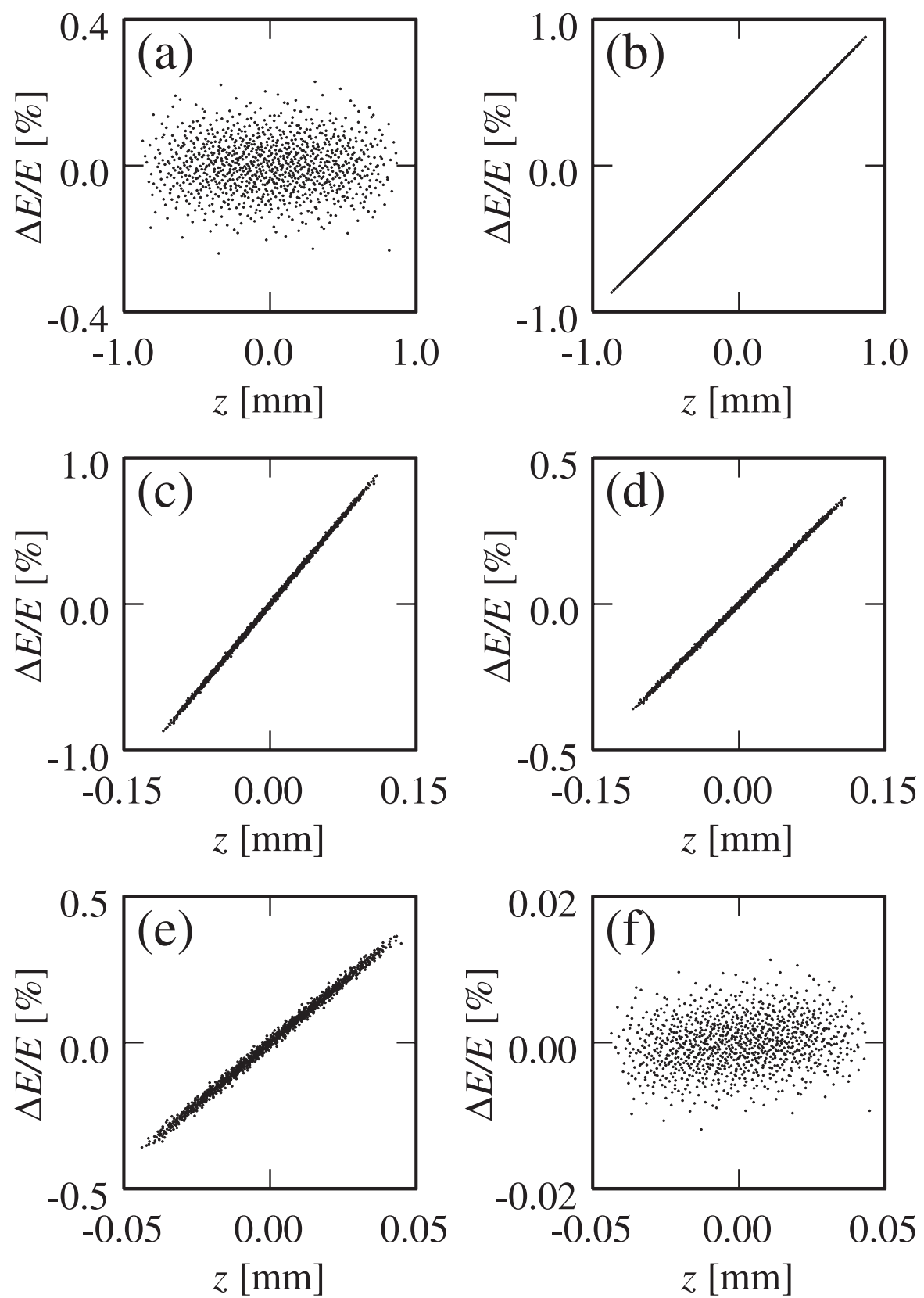

FIG. 2. Bunch compression without collective effects, for an initial parabolic current distribution with rms length of $400 \mu \mathrm{m}$ and 3 $\mathrm{keV}$ Gaussian energy spread, according to ELEGANT simulations of 100000 particles. In the phase-space plots, the longitudinal coordinate $z$ is zero at the average electron position, with $z>0$ at the tail of the bunch. (a) Initial bunch at $4 \mathrm{MeV}$. (b) Chirped bunch at the entrance of the $\mathrm{BC} 1$ chicane. (c) Bunch after single-stage compression in BC1. (d) Chirped bunch at the entrance of the BC2 chicane. (e) Bunch after full compression in BC2. (f) Dechirped bunch at $1.7 \mathrm{GeV}$.

For TESLA 9-cell superconducting cavities, the highfrequency limit of the linac geometric impedance per unit length is given for $k>0$ by [20,21]

$$
Z_{\text {linac }}(k)=\frac{i Z_{0}}{\pi k a^{2}}\left[1+(1+i) \frac{\alpha L_{\text {cell }}}{a}\left(\frac{\pi}{k g}\right)^{1 / 2}\right]^{-1}
$$

where $a=35 \mathrm{~mm}$ is the iris radius and $L_{\text {cell }}=115.4 \mathrm{~mm}$ is the cell period [22], $g=90 \mathrm{~mm}$ is the distance between irises [23], and $\alpha \approx 0.46$ for $g \approx L_{\text {cell }}[21]$.
For a bending magnet in which the orbit's radius of curvature is $\rho$, the coherent radiation builds up over a distance of $\sim \rho^{2 / 3} \lambda^{1 / 3}$ known as the CSR formation length [24]. Provided that the magnet length $L_{b}$ exceeds $\rho^{2 / 3} \lambda^{1 / 3}$, the coherent radiation becomes "fully formed" and approximated by steady-state CSR. The CSR formation length is comparable to the "overtaking length" $(24 / 2 \pi)^{1 / 3} \rho^{2 / 3} \lambda^{1 / 3}$ over which the radiation overtakes the bunch by a distance $\lambda / 2 \pi$ [25-27], indicating that 
the CSR becomes fully formed when it overtakes the modulation that produces it. Downstream of the magnet, the CSR changes to CER over a distance of $\sim \rho^{2 / 3} \lambda^{1 / 3}$. At distance $d$ from the magnet entrance obeying $\rho^{2 / 3} \lambda^{1 / 3}<$ $d<\rho^{2 / 3} \lambda^{1 / 3}+L_{b}$, we approximate the impedance by the impedance per unit length of steady-state CSR, given for $k>0$ by [11]

$$
Z_{\mathrm{CSR}}(k)=1.63 Z_{0}(1+0.58 i) k^{1 / 3} /\left(4 \pi \rho^{2 / 3}\right) .
$$

For typical chicane magnet parameters $\left[L_{b} \sim 0.1 \mathrm{~m}, \rho \sim\right.$ $\left.1 \mathrm{~m}, k \sim 1 / \sigma_{z} \sim 1 /(100 \mu \mathrm{m})\right]$, the integrated CSR impedance over the length $L_{b}$ is $\sim 0.3 Z_{0}(1+0.58 i)$.

Downstream of the magnet, the wake primarily results from coherent edge radiation. For $k>0$, the integrated resistive CER impedance in a drift space of length $L_{d}$ is approximately [14]

$$
\int Z_{\mathrm{CER}}(k)=\frac{Z_{0}}{2 \pi} \ln \left(\frac{\min \left(L_{d}, \lambda \gamma^{2} / 2 \pi\right)}{\rho^{2 / 3} \lambda^{1 / 3}}\right),
$$

for wavelengths where the right-hand side (rhs) of Eq. (4) is positive. For typical chicane magnet parameters $[\gamma \sim$ 1000, $\left.\rho \sim 1 \mathrm{~m}, \lambda / 2 \pi \sim \sigma_{z} \sim 100 \mu \mathrm{m}, L_{d} \sim 10 \mathrm{~m}\right]$, the integrated CER impedance is $\sim Z_{0}$. Because of the weak logarithmic dependence in Eq. (4), an integrated impedance of $\sim Z_{0}$ may be expected for a wide range of parameters.

Approximating a chicane magnet's impedance by CSR and CER regions is realistic when the lengths of the CSR and CER regions $\left[L_{b}\right.$ and $\left.\min \left(L_{d}, \lambda \gamma^{2} / 2 \pi\right)\right]$ exceed the length of the transition between them $\left(\sim \rho^{2 / 3} \lambda^{1 / 3}\right)$. Equation (4) neglects any reduction of the CER impedance from the bunch radius $r_{b}$. This is valid for $\max \left[r_{b}, r_{b}^{2} /(\lambda / 2 \pi)\right] \ll \rho^{2 / 3} \lambda^{1 / 3} \quad$ [14], i.e. $r_{b} \ll$ $\min \left(\rho^{2 / 3} \lambda^{1 / 3}, \rho^{1 / 3} \lambda^{2 / 3}\right)$, which is satisfied by typical chicane magnet parameters $\left[\rho \sim 1 \mathrm{~m}, \quad \lambda / 2 \pi \sim \sigma_{z} \sim\right.$ $\left.100 \mu \mathrm{m}, r_{b} \sim 100 \mu \mathrm{m}\right]$.

For $k<0$, the impedances are determined by $Z(k)=$ $Z^{*}(-k)$. The total impedance is the sum of the contributions given by Eqs. (1)-(4).

\section{B. Effective impedances}

We consider a symmetrical four-magnet chicane whose wakes are dominated by CER in the drift spaces. We represent each impedance within the chicane with effective impedances before and after the chicane. The effective impedances approximate the wake and effect upon compression of the actual impedance when the other impedances in the chicane are neglected. When calculating the effective impedance of the second chicane magnet, we neglect growth in the relative density modulation between the chicane entrance and the second magnet. This is a good approximation in the case of low gain, which describes a typical chicane when the bunch's emittance and energy spread are taken into account [6]. For moderate gain, this is a reasonable approximation for a chicane (such as the WiFEL design) that has a short drift space after the second magnet, resulting in a small integrated CER wake. For this purpose, a symmetrical three-magnet chicane may be regarded as a four-magnet chicane with a zero drift length between the 2nd and 3rd magnets.

Suppose an electron loses energy $\Delta E$ upstream of the $\mathrm{BC} 1$ chicane, so that the deflection in each chicane magnet is increased by $\Delta \theta$. Neglecting the small length of the chicane magnets, its path through the chicane is increased to first order in $\Delta \theta$ by the length $2 L_{1} \theta_{1} \Delta \theta$, where $\theta_{1}$ is the unperturbed deflection in a chicane magnet, and $L_{1}$ is the distance between the 1st and 2 nd chicane magnets. If an electron loses energy $\Delta E$ between the 1st and 2nd chicane magnets, so that the deflection in the final three chicane magnets is increased by $\Delta \theta$, its path changes by the same length. If it loses energy $\Delta E$ between the 2nd and 3rd magnets, so that the deflection in the final two chicane magnets is increased by $\Delta \theta$, its path changes by half as much. Losing energy after the 3rd magnet does not change the path length. Thus, the effect upon bunch compression of an energy loss between the 1st and 2nd chicane magnets is the same as an energy loss upstream of the chicane; the effect of an energy loss between the 2nd and 3rd magnets is the same as an energy loss one-half as large upstream of the chicane, while the effect of an energy loss after the 3rd magnet is the same as that of an energy loss downstream of the chicane.

For a bunch with negligible emittance and energy spread, a current modulation with wave number $k_{0}$ upstream of the chicane, $\Delta I e^{-i k_{0} z}$, results in an equal modulation between the 1st and 2 nd magnets. The effect of the 1st magnet's impedance $Z_{\mathrm{BCl}_{1}}(k)$ upon the wake and bunch compression is given by an equal effective impedance upstream of the chicane. Since the modulation is compressed after exiting the 3rd magnet, we represent the impedances of the $3 \mathrm{rd}$ and 4 th magnets by effective impedances downstream of the chicane equal to $Z_{\mathrm{BCl}_{3}}(k)$ and $Z_{\mathrm{BCl}_{4}}(k)$, respectively.

Between the 2nd and 3rd magnets of a chicane with low gain, the half-compressed current modulation and its wakefield are $\left[2 C_{1} /\left(C_{1}+1\right)\right] \Delta I e^{-i\left[2 C_{1} /\left(C_{1}+1\right)\right] k_{0} z}$ and $\quad\left[2 C_{1} /\left(C_{1}+1\right)\right] Z_{\mathrm{BCl}_{2}}\left\{\left[2 C_{1} /\left(C_{1}+1\right)\right] k_{0}\right\} \times$ $\Delta I e^{-i\left[2 C_{1} /\left(C_{1}+1\right)\right] k_{0} z}$. The effect of the wake upon compression is provided by a wake upstream of the chicane that is half as large. We model this wake with an effective impedance upstream of the chicane entrance given by the func-

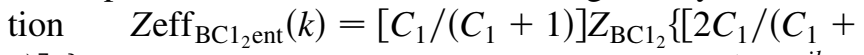
1) $] k\}$, which acts upon the current modulation $\Delta I e^{-i k_{0} z}$. Downstream of a low-gain chicane, the wake between the 2nd and 3rd magnets is compressed to equal $C_{1} Z_{\mathrm{BCl}_{2}}\left\{\left[2 C_{1} /\left(C_{1}+1\right)\right] k_{0}\right\} \Delta I e^{-i C_{1} k_{0} z}$, while the wake from its upstream effective impedance is compressed by the factor $C_{1}$ to give the smaller wake $\left[C_{1}^{2} /\left(C_{1}+\right.\right.$ 1) $] Z_{\mathrm{BCl}_{2}}\left\{\left[2 C_{1} /\left(C_{1}+1\right)\right] k_{0}\right\} \Delta I e^{-i C_{1} k_{0} z}$. By adding an addi- 
tional effective impedance downstream of the chicane exit $\operatorname{Zeff}_{\mathrm{BCl}_{2} \text { exit }}(k)=\left[1 /\left(C_{1}+1\right)\right] Z_{\mathrm{BCl}_{2}}\left\{\left[2 /\left(C_{1}+1\right)\right] k\right\}$, which acts upon the compressed current modulation $C_{1} \Delta I e^{-i C_{1} k_{0} z}$, we approximate the 2 nd magnet's wake and compression.

In a bunch with finite emittance, the amplitude of the modulation is reduced within the chicane by longitudinal smearing [4-6,8]. An electron with horizontal displacement $x$ at the chicane entrance is displaced longitudinally by $x \theta_{1}$ between the $1 \mathrm{st}$ and 2 nd magnets. For a symmetric distribution of horizontal positions $f_{1_{\text {ent }}}(x)$ at the entrance of the chicane, the modulation amplitude and wake after the 1st magnet are modified by the form factor $\int f_{1_{\text {ent }}}(x) \cos \left(k x \theta_{1}\right) d x$, which equals $\exp \left[-\left(k \theta_{1} \sigma_{x 1_{\text {ent }}}\right)^{2} / 2\right]$ for a Gaussian distribution with rms size $\sigma_{x 1_{\text {ent }}}$. The effect of a given wake upon the energy imparted to a longitudinal slice of the incoming bunch is also modified by this form factor. We approximate this emittance effect by modifying the effective impedance of the 1st chicane magnet's CSR and CER by the square of this form factor.

Similarly, an electron with horizontal displacement $x$ at the chicane exit is displaced longitudinally by $-x \theta_{1}$ between the 3rd and 4th magnets. For a symmetric distribution of horizontal positions $f_{1_{\text {exit }}}(x)$ at the exit of the chicane, the modulation amplitude and wake after the 3rd magnet are modified by the form factor $\int f_{1_{\text {exit }}}(x) \times$ $\cos \left(k x \theta_{1}\right) d x$, which equals $\exp \left[-\left(k \theta_{1} \sigma_{x 1_{\text {exit }}}\right)^{2} / 2\right]$ for a Gaussian distribution with rms size $\sigma_{x 1_{\text {exit }}}$. The effect of a given wake upon the energy imparted to a longitudinal slice of the outgoing bunch is also modified by this form factor. We approximate this emittance effect by modifying the effective impedance of the 3rd magnet's CSR and CER by the square of this form factor.

An electron with horizontal angle $x^{\prime}$ is displaced longitudinally by $-x^{\prime} L_{1} \theta_{1}$ between the 2 nd and 3rd magnets. For a symmetric distribution of horizontal angle $f_{1}^{\prime}\left(x^{\prime}\right)$ at the entrance of the chicane, the modulation amplitude and wake of the 2 nd magnet are modified by the form factor $\int f_{1}^{\prime}\left(x^{\prime}\right) \cos \left\{\left[2 C_{1} /\left(C_{1}+1\right)\right] k_{0} x^{\prime} L_{1} \theta_{1}\right\} d x^{\prime}$ for a modulation with wave number $k_{0}$ upstream of the chicane. This form factor equals $\exp \left(-\left\{\left[2 C_{1} /\left(C_{1}+1\right)\right] k_{0} L_{1} \theta_{1} \sigma_{x 1}^{\prime}\right\}^{2} / 2\right)$ for a Gaussian distribution with rms divergence $\sigma_{x 1}^{\prime}$. The effect of a given wake upon the energy imparted to a longitudinal slice of the incoming bunch is also modified by this form factor. We approximate this effect by modifying the effective impedances of the 2nd chicane magnet's CSR and CER located upstream and downstream of the chicane. The effective impedance upstream of the chicane is modified by the square of the form factor $\int f_{1}^{\prime}\left(x^{\prime}\right) \times$ $\cos \left\{\left[2 C_{1} /\left(C_{1}+1\right)\right] k x^{\prime} L_{1} \theta_{1}\right\} d x^{\prime}$; where the form factor equals $\exp \left(-\left\{\left[2 C_{1} /\left(C_{1}+1\right)\right] k L_{1} \theta_{1} \sigma_{x 1}^{\prime}\right\}^{2} / 2\right)$ for a Gaussian distribution with rms divergence $\sigma_{x 1}^{\prime}$. The 2nd magnet's effective impedance downstream of the chicane is modified by the square of the form factor $\int f_{1}^{\prime}\left(x^{\prime}\right) \times$ $\cos \left\{\left[2 /\left(C_{1}+1\right)\right] k x^{\prime} L_{1} \theta_{1}\right\} d x^{\prime}$, in which we have accounted for the fact that the downstream modulation has wave number $k=C_{1} k_{0}$. This form factor equals $\exp \left(-\left\{\left[2 /\left(C_{1}+1\right)\right] k L_{1} \theta_{1} \sigma_{x 1}^{\prime}\right\}^{2} / 2\right)$ for a Gaussian distribution with rms divergence $\sigma_{x 1}^{\prime}$.

A similar analysis applies to the second chicane $\mathrm{BC} 2$, in which $\theta_{2}$ is the unperturbed deflection, $L_{2}$ is the distance between the first and second magnets, and the bunches have rms divergence $\sigma_{x 2}^{\prime}$ and horizontal sizes $\sigma_{x 2_{\text {ent }}}$ and $\sigma_{x 2_{\text {exit }}}$ at the entrance and exit.

For Gaussian distributions of horizontal position and angle, we therefore represent the impedances of the first two magnets of $\mathrm{BC} 1$ with effective impedances upstream of the chicane entrance equaling

$$
\begin{aligned}
\operatorname{Zeff}_{\mathrm{BCl}_{1}}(k)= & Z_{\mathrm{BCl}_{1}}(k) \exp \left(-\left[k \theta_{1} \sigma_{x 1_{\text {ent }}}\right]^{2}\right) \\
\operatorname{Zeff}_{\mathrm{BCl}_{2} \text { ent }}(k)= & \frac{C_{1}}{C_{1}+1} Z_{\mathrm{BCl}_{2}}\left(\frac{2 C_{1} k}{C_{1}+1}\right) \\
& \times \exp \left(-\left[\frac{2 C_{1} k L_{1} \theta_{1} \sigma_{x 1}^{\prime}}{C_{1}+1}\right]^{2}\right),
\end{aligned}
$$

where $Z_{\mathrm{BCl}_{1}}(k)$ and $Z_{\mathrm{BCl}_{2}}(k)$ are impedances of the first two magnets of BC1 from CSR or CER. For a modulation with initial wavelength $\lambda_{0}$ and initial wave number $k_{0}$, effective impedances of WiFEL are shown in Fig. 3(a). The total effective impedance $Z_{\text {zero }}(k)$, which acts upon the bunch before it is compressed by $\mathrm{BC} 1$, is the sum of the effective CSR and CER impedances of the first two magnets of BC1. For initial wavelengths less than $400 \mu \mathrm{m}$, the effective impedance $Z_{\text {zero }}(k)$ is suppressed by the bunch's emittance.

Between $\mathrm{BC} 1$ and $\mathrm{BC} 2$, we have the effective impedances

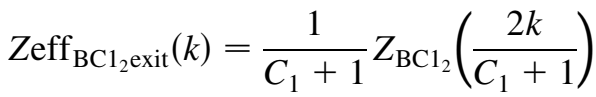

$$
\begin{aligned}
& \times \exp \left(-\left[\frac{2 k L_{1} \theta_{1} \sigma_{x 1}^{\prime}}{C_{1}+1}\right]^{2}\right), \\
& Z_{\operatorname{eff}} \mathrm{BCl}_{3}(k)=Z_{\mathrm{BCl}_{3}}(k) \exp \left(-\left[k \theta_{1} \sigma_{x 1_{\text {exit }}}\right]^{2}\right) \\
& Z_{\text {eff }} \mathrm{BCl}_{4}(k)=Z_{\mathrm{BCl}_{4}}(k) \\
& Z \operatorname{eff}_{\mathrm{BC}_{2}{ }_{1}}(k)=Z_{\mathrm{BC}_{1}}(k) \exp \left(-\left[k \theta_{2} \sigma_{x 2_{\text {ent }}}\right]^{2}\right) \\
& Z_{\text {eff }}{\mathrm{BC} 22_{2} \text { ent }}(k)=\frac{C_{2}}{C_{2}+1} Z_{\mathrm{BC}_{2}}\left(\frac{2 C_{2} k}{C_{2}+1}\right) \\
& \times \exp \left(-\left[\frac{2 C_{2} k L_{2} \theta_{2} \sigma_{x 2}^{\prime}}{C_{2}+1}\right]^{2}\right) \text {, }
\end{aligned}
$$

where the impedances on the rhs result from CSR or CER. The effective impedance $Z_{1}(k)$, which acts upon the bunch between $\mathrm{BC} 1$ and $\mathrm{BC} 2$, is given by summing these effective CSR and CER impedances with the LSC and linac geometric impedances of the L1 linac. For a modulation with initial wavelength $\lambda_{0}$, the effective impedances experienced between $\mathrm{BC} 1$ and $\mathrm{BC} 2$ are shown in Fig. 3(b). The LSC impedance and the linac geometric impedance are 

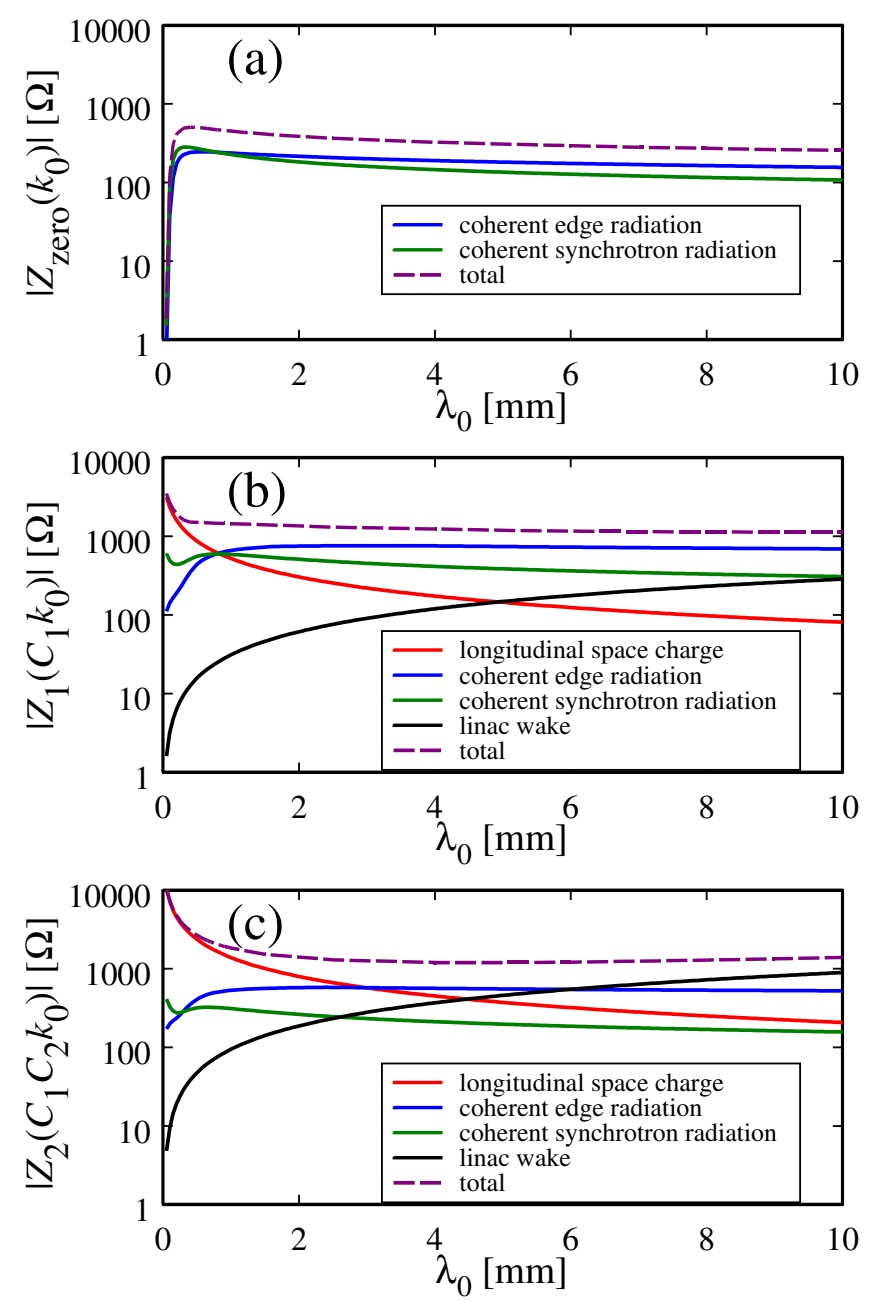

FIG. 3. (Color) Effective impedances experienced by a modulation whose wavelength and wave number before compression are $\lambda_{0}$ and $k_{0}$, respectively. (a) Impedance experienced before compression in $\mathrm{BC} 1$. (b) Impedance experienced between $\mathrm{BC} 1$ and $\mathrm{BC} 2$. (c) Impedance experienced after full compression in $\mathrm{BC} 2$.

evaluated for a bunch with normalized horizontal emittance and vertical emittance each equaling $1 \mu \mathrm{m}$-rad, accelerated from 215 to $485 \mathrm{MeV}$ at a constant rate over the 16.6-m length of the L1 linac, in which the traditional noncanonical beta functions [28] obey $\left\langle\beta_{x}\right\rangle \approx\left\langle\beta_{y}\right\rangle \approx$ $25 \mathrm{~m}$. The total effective impedance experienced by the modulation is $Z_{1}\left(C_{1} k_{0}\right)$.

We represent the remaining impedances of $\mathrm{BC} 2$ with effective impedances downstream of $\mathrm{BC} 2$ from CSR and CER

$$
\begin{aligned}
& \operatorname{Zeff}_{\mathrm{BC}_{2} \text { exit }}(k)=\frac{1}{C_{2}+1} Z_{\mathrm{BC}_{2}}\left(\frac{2 k}{C_{2}+1}\right) \\
& \times \exp \left(-\left[\frac{2 k L_{2} \theta_{2} \sigma_{x 2}^{\prime}}{C_{2}+1}\right]^{2}\right), \\
& Z_{\operatorname{eff}}{\mathrm{BC} 2_{3}}_{3}(k)=Z_{\mathrm{BC}_{3}}(k) \exp \left(-\left[k \theta_{2} \sigma_{x 2_{\text {exit }}}\right]^{2}\right) \\
& Z_{\operatorname{eff}} \mathrm{BC} 2_{4}(k)=Z_{\mathrm{BC}_{4}}(k) \text {. }
\end{aligned}
$$

The total effective impedance $Z_{2}(k)$ downstream of $\mathrm{BC} 2$ is given by summing the effective CSR and CER impedances with the LSC and linac geometric impedances of the L2 linac. For a modulation with initial wavelength $\lambda_{0}$, the effective impedances experienced after BC2 are shown in Fig. 3(c). The LSC impedance and the linac geometric impedance are evaluated for a bunch with normalized horizontal and vertical emittances of $1 \mu \mathrm{m}$-rad that is accelerated from $485 \mathrm{MeV}$ to $1.7 \mathrm{GeV}$ at a constant rate in the L2 linac of length $124.5 \mathrm{~m}$, where $\left\langle\beta_{x}\right\rangle \approx\left\langle\beta_{y}\right\rangle \approx$ $25 \mathrm{~m}$. The length of BC2's final two magnets is smaller than the steady-state CSR formation length for $\lambda_{0}>$ $3 \mathrm{~mm}$. For $\lambda_{0}>3 \mathrm{~mm}$, where the CSR impedance gives a negligible contribution to the total impedance, the CSR estimate becomes inaccurate; an accurate estimate would require consideration of the wake of a short magnet [2527]. The total effective impedance experienced after $\mathrm{BC} 2$ is $Z_{2}\left(C_{1} C_{2} k_{0}\right)$. Since the bunch's longitudinal particle distribution is effectively frozen after the $\mathrm{BC} 2$ chicane, the impedance $Z_{2}$ affects the bunch's energy distribution, but not its current profile.

The impedances before and after $\mathrm{BC} 1$ are dominated by the resistive impedance of CER for initial wavelengths exceeding $1 \mathrm{~mm}$. Since a bunch with initial $\mathrm{rms}$ bunch length $\sigma_{z}$ is primarily composed of wavelengths exceeding $2 \pi \sigma_{z}$, the impedances before and after $\mathrm{BC} 1$ are dominated by CER for $2 \pi \sigma_{z}>1 \mathrm{~mm}$, i.e. $\sigma_{z}>160 \mu \mathrm{m}$. For short wavelengths, the LSC impedance dominates.

Since we are planning for WiFEL initial bunch lengths of $\sim 400 \mu \mathrm{m}$, we expect that macroscopic wake effects before and after $\mathrm{BC} 1$ will be dominated by the resistive impedance of CER.

\section{MACROSCOPIC MODELING WITH RESISTIVE IMPEDANCE}

\section{A. Tracking simulations}

To study the approximation of wakes by resistive effective impedances, we performed tracking simulations. Numerical noise was reduced by sampling the initial phase space with Halton sequences [7,29]. To isolate the effect of coherent radiation, LSC and linac geometric wakes are not included in these initial simulations.

We performed longitudinal tracking with a modified version of the code LITRACK [30], in which impedances $Z_{\text {zero }}(k), Z_{1}(k)$, and $Z_{2}(k)$ (calculated without LSC and linac impedances) are approximated by resistances $R_{0}$, $R_{1}$, and $R_{2}$. To model initial bunch lengths in the range of $400 \mu \mathrm{m}$ to $1 \mathrm{~mm}$, we averaged the values of $\left|Z_{\text {zero }}\left(k_{0}\right)\right|$, $\left|Z_{1}\left(C_{1} k_{0}\right)\right|$, and $\left|Z_{2}\left(C_{1} C_{2} k_{0}\right)\right|$ for initial wavelengths of $2 \pi \times 400 \mu \mathrm{m}$ and $2 \pi \times 1 \mathrm{~mm}$ (i.e., 2.5 and $6.28 \mathrm{~mm}$ ), obtaining resistances $R_{0}, R_{1}$, and $R_{2}$ of 327, 1121, and $756 \Omega$, respectively. Since a drift length of several meters has resistive CER impedance of $\sim Z_{0}$, and the WiFEL chicanes have much shorter drift lengths after their second magnets, $R_{0} \sim Z_{0}, R_{1} \sim 3 Z_{0}$, and $R_{2} \sim 2 Z_{0}$. These approximate longitudinal simulations are compared with 
tracking by the ELEGANT code [17], which tracks a sixdimensional phase space.

Tracking without wakes was performed for a trapezoidal bunch of 100000 particles with flattop of $2 \mathrm{~mm}$, peak current of $50 \mathrm{~A}$, Gaussian energy spread of $3 \mathrm{keV}$, and normalized transverse emittances of $1 \mu \mathrm{m}$-rad. At the head and tail, the bunch current drops linearly to zero over a distance of $1 \mathrm{~mm}$. Figure 4(a) shows the longitudinal phase space at $1.7 \mathrm{GeV}$ according to the longitudinal tracking code LITRACK and the ELEGANT code. To ensure that the bunch entering the $\mathrm{BC} 1$ compressor is the same with both codes, we begin tracking at $215 \mathrm{MeV}$, where the bunch's energy profile is the sum of the 4-MeV electron-gun energy with the rf fields of the injector linac and harmonic cavities.

The output phase spaces agree within $\Delta E / E=0.01 \%$. The bunch is compressed by a factor of 20 to a peak current of $1 \mathrm{kA}$; the compressed bunch's flattop current is constant within $5 \%$.

Figure 4(b) shows the fully compressed trapezoidal bunch at $1.7 \mathrm{GeV}$, tracked by LITRACK with resistive impedances $R_{0}, R_{1}$, and $R_{2}$. The compressed bunch forms three line segments in phase space, in which the middle line segment is horizontal.

Figure 4(b) also shows an ELEGANT simulation where longitudinal coherent-radiation wakes are applied in all of the chicane bending magnets and drift regions downstream of them. The longitudinal coherent-radiation wakes in the magnets and downstream drift regions are computed by ELEGANT in the $\gamma \rightarrow \infty$ limit, according to the method of Ref. [27]. Transient effects at the beginning of the magnets and drift regions are included. We expect this method to provide an accurate calculation of CER for wavelengths where $r_{b} \ll \min \left(\rho^{2 / 3} \lambda^{1 / 3}, \rho^{1 / 3} \lambda^{2 / 3}\right)$, provided that the drift region length $L_{d}$ does not greatly exceed the edgeradiation formation length of $\lambda \gamma^{2} / 2 \pi$ [14]. These requirements are satisfied for wavelengths where the calculated impedance is dominated by CER. Shielding of the wakes by the vacuum chamber is not modeled.

Figure 4(c) shows LITRACK and ELEGANT tracking of a Gaussian bunch with peak current of $50 \mathrm{~A}$ and rms bunch length of $1 \mathrm{~mm}$, while Fig. 4(d) shows tracking of a parabolic bunch with peak current of $50 \mathrm{~A}$ and rms bunch length of $1 \mathrm{~mm}$. In all cases, an initial Gaussian energy spread of $3 \mathrm{keV}$ and normalized transverse emittances of $1 \mu \mathrm{m}$-rad are modeled.

According to ELEGANT tracking, the CSR and CER wakes increase the horizontal projected and slice emittances at $1.7 \mathrm{GeV}$ by $\sim 30 \%$ and $\sim 10 \%$, respectively, while shifting the bunch centroid horizontally by $\sim 100 \mu \mathrm{m}$ in position and $\sim 5 \mu \mathrm{rad}$ in angle. These shifts, which are comparable to the bunch's rms size and divergence, can be compensated by steering magnets.

The similarity of the longitudinal tracking with LITRACK and ELEGANT confirms that the macroscopic effects of coherent radiation can be approximated by resistive effective impedances. When the geometric wakes of the L1 and L2 linacs are also included in the tracking, the only significant difference is a linear chirp of the bunch at $1.7 \mathrm{GeV}$. This is consistent with Fig. 3, in which CER is the dominant impedance for wavelengths comparable to $2 \pi$ times the bunch length.

\section{B. Upright bunches in phase space}

In Appendix A, we consider the passage of a cold bunch through a two-stage compressor with resistive impedances $R_{0}, R_{1}$, and $R_{2}$. The longitudinal coordinates of an electron after each chicane, $z_{1}$ and $z_{2}$, are related to the initial coordinate $z$. Since the longitudinal particle distribution is effectively frozen after $\mathrm{BC} 2$, the relation $z_{2}(z)$ applies to WiFEL when the impedances $Z_{\text {zero }}(k)$ and $Z_{1}(k)$ are approximately resistive: for $\sigma_{z}>160 \mu \mathrm{m}$.

For a bunch with initial current magnitude $I(z)$, whose initial energy profile differs from a linear chirp by $\Delta E(z)$, the longitudinal coordinate after $\mathrm{BC} 1$ obeys

$$
z_{1}(z)=z / C_{1}+\left(R_{56}^{(1)} / E_{1}\right)\left[\Delta E(z)-e R_{0} I(z)\right],
$$

with derivative with respect to $z$ of

$$
z_{1}^{\prime}(z)=C_{1}^{-1}\left\{1+C_{1}\left(R_{56}^{(1)} / E_{1}\right)\left[\Delta E^{\prime}(z)-e R_{0} I^{\prime}(z)\right]\right\},
$$

where $e>0$ is the magnitude of the electron charge.

When the rhs of Eq. (9) is zero, an upright bunch occurs after $\mathrm{BC} 1$. Thus, part of the bunch is upright after $\mathrm{BC} 1$ when

$$
\left(-C_{1} R_{56}^{(1)} / E_{1}\right)\left[\Delta E^{\prime}(z)-e R_{0} I^{\prime}(z)\right]=1 .
$$

When none of the bunch is upright after $\mathrm{BC} 1$, the longitudinal coordinate after $\mathrm{BC} 2$ is

$$
z_{2}(z)=\frac{1}{C_{1} C_{2}}\left\{z+\left(\frac{C_{1} R_{56}^{(1)}}{E_{1}}+\frac{C_{1}^{2} C_{2} R_{56}^{(2)}}{E_{2}}\right)\left[\Delta E(z)-e R_{0} I(z)\right]-\frac{C_{1}^{2} C_{2}\left(R_{56}^{(2)} / E_{2}\right) e R_{1} I(z)}{1+C_{1}\left(R_{56}^{(1)} / E_{1}\right)\left[\Delta E^{\prime}(z)-e R_{0} I^{\prime}(z)\right]}\right\}
$$

with derivative

$$
\begin{aligned}
z_{2}^{\prime}(z)= & \frac{1}{C_{1} C_{2}}\left\{1+\left(\frac{C_{1} R_{56}^{(1)}}{E_{1}}+\frac{C_{1}^{2} C_{2} R_{56}^{(2)}}{E_{2}}\right)\left[\Delta E^{\prime}(z)-e R_{0} I^{\prime}(z)\right]-\frac{C_{1}^{2} C_{2}\left(R_{56}^{(2)} / E_{2}\right) e R_{1} I^{\prime}(z)}{1+C_{1}\left(R_{56}^{(1)} / E_{1}\right)\left[\Delta E^{\prime}(z)-e R_{0} I^{\prime}(z)\right]}\right. \\
& \left.+\frac{C_{1}^{3} C_{2}\left(R_{56}^{(1)} / E_{1}\right)\left(R_{56}^{(2)} / E_{2}\right) e R_{1} I(z)\left[\Delta E^{\prime \prime}(z)-e R_{0} I^{\prime \prime}(z)\right]}{\left\{1+C_{1}\left(R_{56}^{(1)} / E_{1}\right)\left[\Delta E^{\prime}(z)-e R_{0} I^{\prime}(z)\right]\right\}^{2}}\right\} .
\end{aligned}
$$



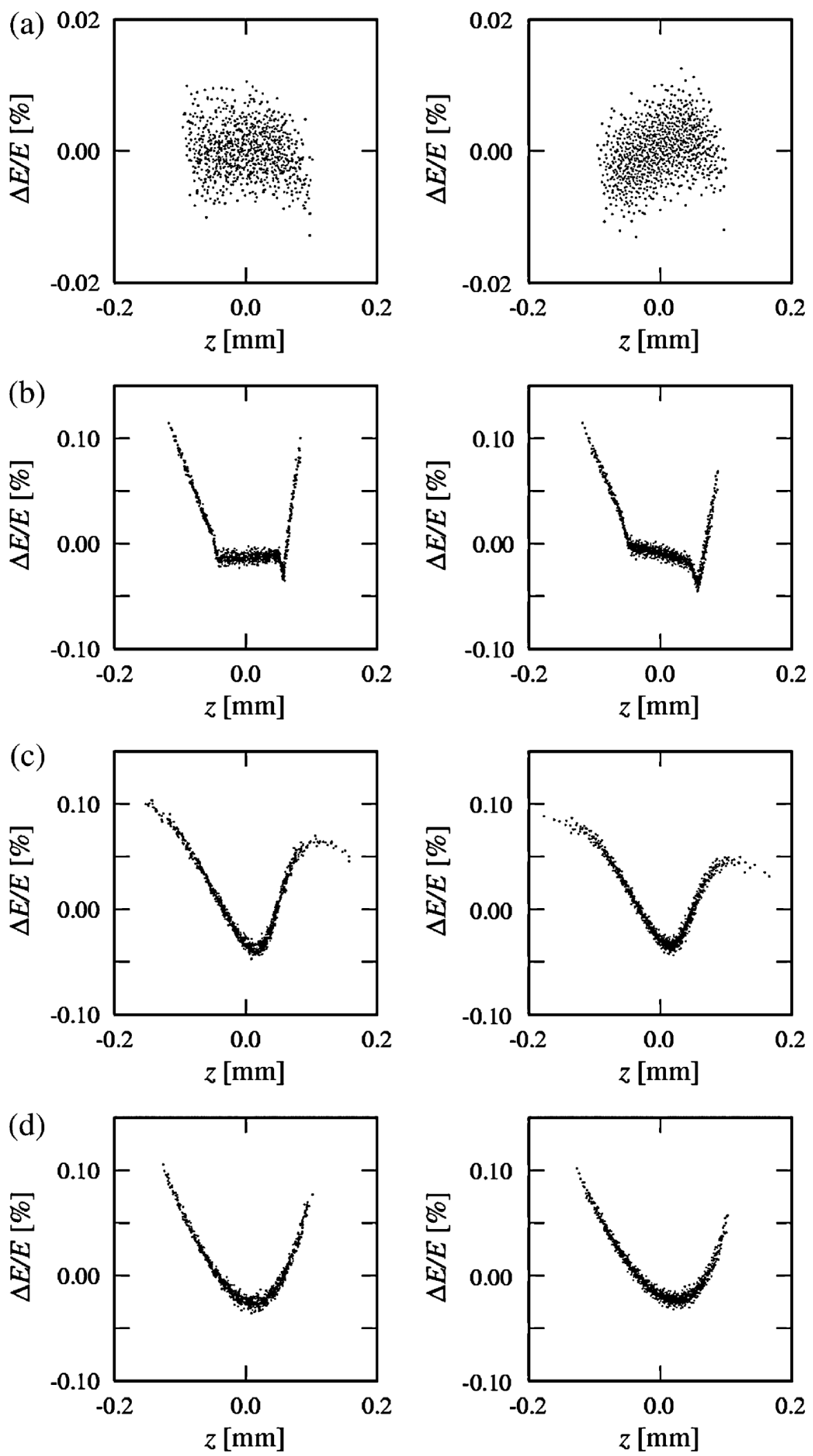

FIG. 4. Phase space after compression and acceleration to $1.7 \mathrm{GeV}$, for bunches with peak current of 50 A before compression. The bunch tail is at positive $z$. LITRACK (with resistive impedance) results are on the left, while ELEGANT results with coherent radiation are on the right. (a) Trapezoidal bunch with 2-mm flattop, 1-mm head and tail, tracked without collective effects. (b) Trapezoidal bunch with 2-mm flattop, 1-mm head and tail. (c) Gaussian bunch with $\sigma_{z}=1 \mathrm{~mm}$. (d) Parabolic bunch with $\sigma_{z}=1 \mathrm{~mm}$. 
When $z_{2}^{\prime}(z)=0$, part of the bunch is upright in phase space after BC2.

When $\left|\left(C_{1} R_{56}^{(1)} / E_{1}\right)\left[\Delta E^{\prime}(z)-e R_{0} I^{\prime}(z)\right]\right| \ll 1$ for all $z$, upright bunch behavior after BC1 is safely avoided, and Eq. (12) may be approximated as

$$
\begin{aligned}
z_{2}^{\prime}(z) \approx & \frac{1}{C_{1} C_{2}}\left\{1+\left(\frac{C_{1} R_{56}^{(1)}}{E_{1}}+\frac{C_{1}^{2} C_{2} R_{56}^{(2)}}{E_{2}}\right)\right. \\
& \left.\times\left[\Delta E^{\prime}(z)-e R_{0} I^{\prime}(z)\right]-\frac{C_{1}^{2} C_{2} R_{56}^{(2)}}{E_{2}} e R_{1} I^{\prime}(z)\right\},
\end{aligned}
$$

provided that $I(z)$ and $\Delta E(z)$ do not vary on a length scale much shorter than the bunch.

For a bunch with a linear chirp $[\Delta E(z)=0]$, Eq. (13) predicts upright bunch behavior for

$I^{\prime}(z)=\left[\left(\frac{C_{1} R_{56}^{(1)}}{E_{1}}+\frac{C_{1}^{2} C_{2} R_{56}^{(2)}}{E_{2}}\right) e R_{0}+\left(\frac{C_{1}^{2} C_{2} R_{56}^{(2)}}{E_{2}}\right) e R_{1}\right]^{-1}$.

When $\Delta E(z)=e R_{0} I(z)$, the effect of the resistance $R_{0}$ is perfectly compensated to achieve linear compression in $\mathrm{BC}$. In this idealized case, upright bunch behavior is predicted by Eq. (13) for

$$
I^{\prime}(z)=E_{2} /\left(C_{1}^{2} C_{2} R_{56}^{(2)} e R_{1}\right) .
$$

\section{Minimum initial bunch length}

Consider a bunch with peak current magnitude $I_{0}$ that is compressed to a peak current $C_{1} C_{2} I_{0}$. In this section we show that the compressed bunch may have an upright tail region in phase space and an associated current spike if the initial uncompressed bunch is too short. We consider the case where $\left|\left(C_{1} R_{56}^{(1)} / E_{1}\right)\left[\Delta E^{\prime}(z)-e R_{0} I^{\prime}(z)\right]\right| \ll 1$, so that upright bunch behavior after $\mathrm{BC} 1$ is safely avoided.

For a linearly chirped trapezoidal initial bunch, in which the current at the head and tail drop linearly to zero over a distance $\Delta z$, Eq. (14) indicates that the tail of the compressed bunch will be upright in phase space after $\mathrm{BC} 2$ when

$$
\begin{aligned}
\Delta z & =\Delta z_{\text {crit }} \\
& =\left|\left(\frac{C_{1} R_{56}^{(1)}}{E_{1}}+\frac{C_{1}^{2} C_{2} R_{56}^{(2)}}{E_{2}}\right) e R_{0}+\left(\frac{C_{1}^{2} C_{2} R_{56}^{(2)}}{E_{2}}\right) e R_{1}\right| I_{0} .
\end{aligned}
$$

For $I_{0}=50 \mathrm{~A}, \Delta z_{\text {crit }}=480 \mu \mathrm{m}$ is predicted for WiFEL. The upright bunch tail predicted for $\Delta z=\Delta z_{\text {crit }}$ occurs in the LITRACK and ELEGANT simulations of Fig. 5 (a) performed for normalized transverse emittances of $1 \mu \mathrm{m}$-rad.

If the effect of $R_{0}$ could be perfectly compensated to give linear compression in $\mathrm{BC} 1$, Eq. (15) predicts an up- right tail would occur for

$$
\Delta z=\Delta z_{\text {crit }}=\left|C_{1}^{2} C_{2} R_{56}^{(2)} e R_{1} I_{0} / E_{2}\right| .
$$

For perfect compensation, which is impractical for the tails of a trapezoidal bunch, $\Delta z_{\text {crit }}$ becomes $330 \mu \mathrm{m}$.

For a Gaussian initial bunch with peak current $I_{0}, I(z)=$ $I_{0} e^{-z^{2} / 2 \sigma_{z}^{2}}$. An upright tail region is predicted for a linear chirp by Eq. (14) when

$$
\begin{aligned}
\sigma_{z} \leq & \sigma_{z_{\text {crit }}} \\
= & e^{-1 / 2} \mid\left(\frac{C_{1} R_{56}^{(1)}}{E_{1}}+\frac{C_{1}^{2} C_{2} R_{56}^{(2)}}{E_{2}}\right) e R_{0} \\
& +\left(\frac{C_{1}^{2} C_{2} R_{56}^{(2)}}{E_{2}}\right) e R_{1} \mid I_{0} .
\end{aligned}
$$

For $I_{0}=50 \mathrm{~A}, \sigma_{z_{\text {crit }}}$ equals $290 \mu \mathrm{m}$. Gaussian bunches tracked in Fig. 5(b) show upright bunch tails for $\sigma_{z}=$ $\sigma_{z_{\text {crit }}}$.

If the effect of $R_{0}$ could be perfectly compensated to give linear compression in BC1, Eq. (15) predicts an upright tail for

$$
\sigma_{z} \leq \sigma_{z_{\text {crit }}}=e^{-1 / 2}\left|C_{1}^{2} C_{2} R_{56}^{(2)} e R_{1} I_{0} / E_{2}\right|
$$

which equals $200 \mu \mathrm{m}$.

For a parabolic initial bunch with peak current $I_{0}, I(z)=$ $I_{0}\left[1-z^{2} /\left(5 \sigma_{z}^{2}\right)\right]$. An upright tail region is predicted for a linear chirp by Eq. (14) when

$$
\begin{aligned}
\sigma_{z} \leq & \sigma_{z_{\text {crit }}} \\
= & \frac{2}{\sqrt{5}} \mid\left(\frac{C_{1} R_{56}^{(1)}}{E_{1}}+\frac{C_{1}^{2} C_{2} R_{56}^{(2)}}{E_{2}}\right) e R_{0} \\
& +\left(\frac{C_{1}^{2} C_{2} R_{56}^{(2)}}{E_{2}}\right) e R_{1} \mid I_{0} .
\end{aligned}
$$

For $I_{0}=50 \mathrm{~A}, \sigma_{z_{\text {crit }}}$ equals $430 \mu \mathrm{m}$. The parabolic bunches tracked in Fig. 5(c) show upright bunch tails and current spikes for $\sigma_{z}=\sigma_{z_{\text {citi }}}$.

If the effect of $R_{0}$ could be perfectly compensated to give linear compression in BC1, Eq. (15) predicts an upright tail for

$$
\sigma_{z} \leq \sigma_{z_{\text {crit }}}=(2 / \sqrt{5})\left|C_{1}^{2} C_{2} R_{56}^{(2)} e R_{1} I_{0} / E_{2}\right|,
$$

which equals $300 \mu \mathrm{m}$. With perfect compensation of $R_{0}$, the bunch length may be decreased $30 \%$ without obtaining an upright tail after $\mathrm{BC} 2$.

\section{Wake compensation}

In WiFEL, we plan to compress a 200 -pC parabolic bunch with peak current of $50 \mathrm{~A}$, rms bunch length of $400 \mu \mathrm{m}$, and normalized transverse emittances of $1 \mu \mathrm{m}$-rad. Figure 5(c) indicates that the compressed bunch 

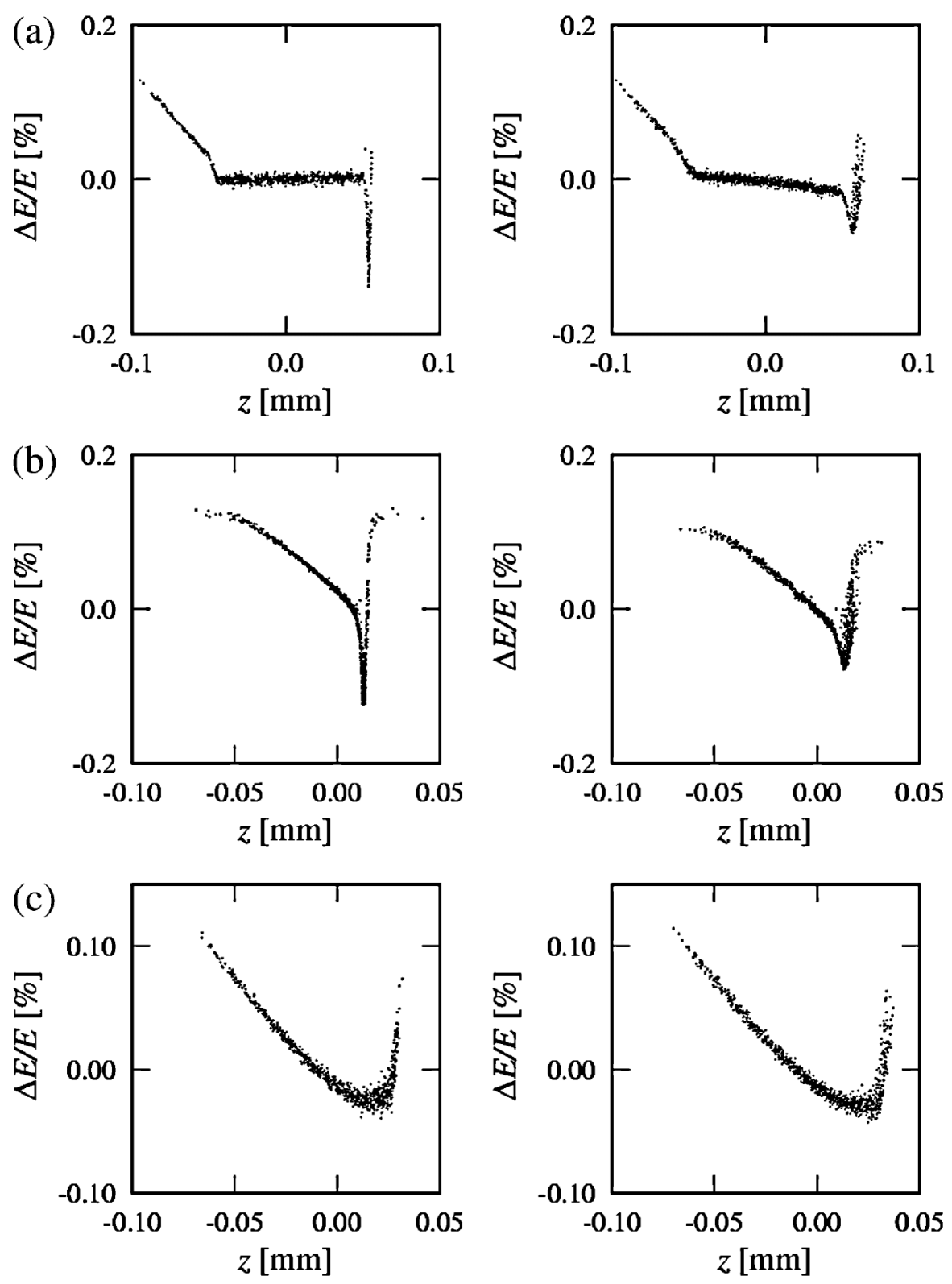

FIG. 5. Phase space at $1.7 \mathrm{GeV}$ showing upright bunch tails for bunches with peak current of 50 A and Gaussian energy spread of $3 \mathrm{keV}$ before compression. LITRACK (with resistive impedance) results are on the left; ELEGANT results with coherent radiation are on the right. (a) Trapezoidal bunch with 2-mm flattop, 480- $\mu \mathrm{m}$ head and tail. (b) Gaussian bunch with $\sigma_{z}=290 \mu \mathrm{m}$. (c) Parabolic bunch with $\sigma_{z}=430 \mu \mathrm{m}$.

will have an upright tail if wake effects are not compensated. For a parabolic bunch, the resistive impedance $R_{0}$ can be compensated by reducing the harmonic cavity voltage so that the rf curvature provides a nonlinear (quadratic) chirp at the entrance of $\mathrm{BC} 1$, in addition to the approximately linear chirp.

We adjusted the rf parameters by longitudinal tracking with a modified version of LITRACK that applies resistive impedances $R_{0}, R_{1}$, and $R_{2}$. The chicane parameters and energies were not changed. With the quick turnaround time of LITRACK, acceptable compensation is quickly found by trial and error. A LITRACK simulation of the compressed bunch at $1.7 \mathrm{GeV}$ is shown in Fig. 6(a). According to
LITRACK, the adjusted rf parameters provide good compression in which the energy profile has a minimum when the bunch current peaks at $1 \mathrm{kA}$. This behavior is nearly reproduced in Fig. 6(a) when 6D tracking from $215 \mathrm{MeV}$ to $1.7 \mathrm{GeV}$ is performed with coherent radiation by the ELEGANT code, using the rf parameters obtained from the LITRACK simulations.

When the geometric wakes of the L1 and L2 linacs are also included in the LITRACK model, adjusting the phases of the $\mathrm{L} 1$ and $\mathrm{L} 2$ linacs by trial and error gives the compressed bunch shown in Fig. 6(b). Figure 6(b) also shows tracking by ELEGANT in which coherent radiation and the linac geometric wakes of L1 and L2 are included. The 

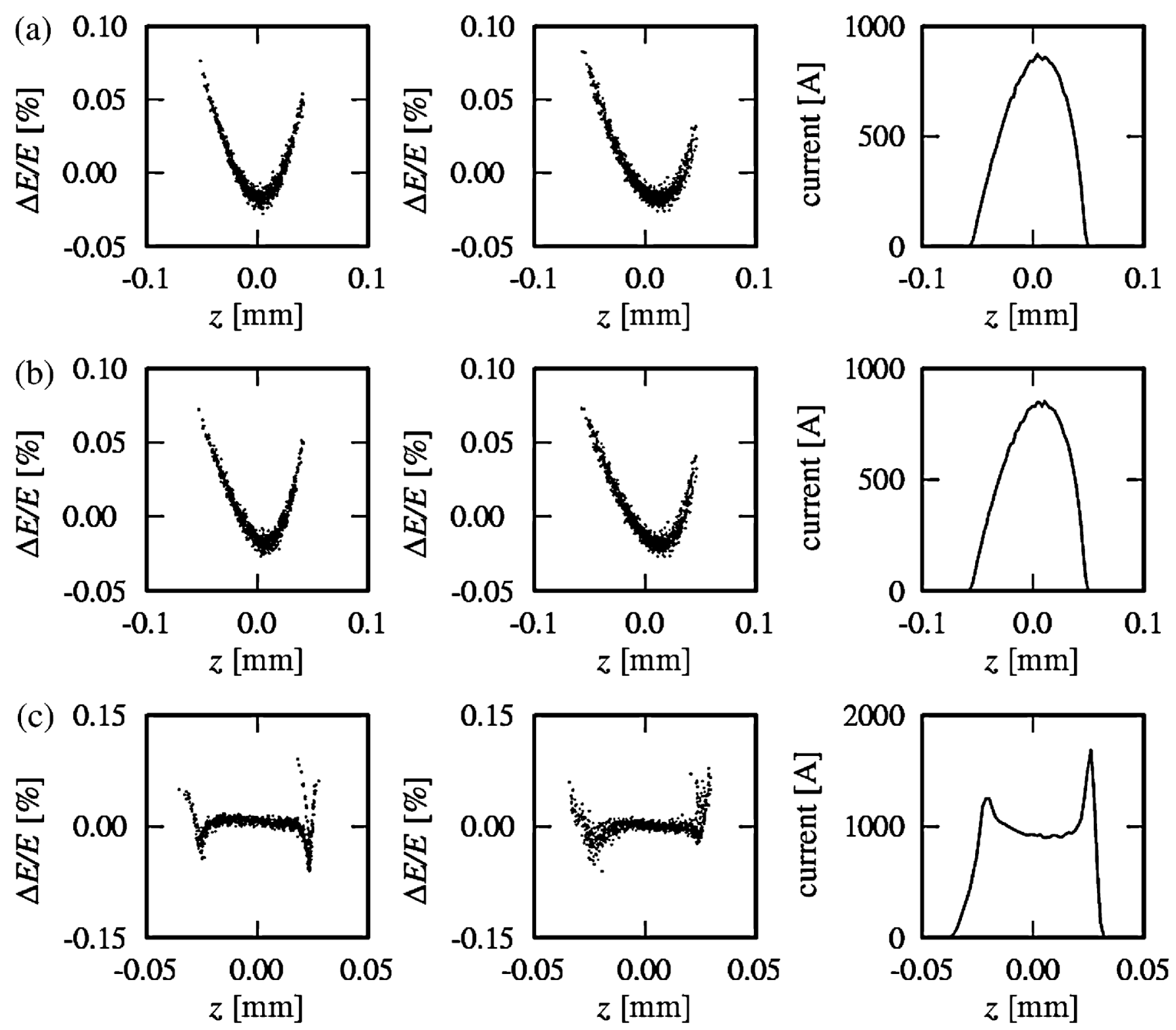

FIG. 6. Compensation of wake effects for an initial parabolic bunch with peak current of $50 \mathrm{~A}, \sigma_{z}=400 \mu \mathrm{m}$, and Gaussian energy spread of $3 \mathrm{keV}$. The left-hand plots show the longitudinal phase space at $1.7 \mathrm{GeV}$ according to LITRACK (with resistive impedance approximation of chicane wakes). The middle plots show the longitudinal phase space at $1.7 \mathrm{GeV}$ according to ELEGANT. The righthand plots show the current distribution at $1.7 \mathrm{GeV}$ according to ELEGANT. The bunch tail is at positive $z$. (a) CSR and CER wakes. (b) CSR, CER, linac L1, and linac L2 wakes. (c) CSR, CER, linac L1, linac L2, injector linac, and harmonic cavity wakes.

ELEGANT tracking confirms that good compression is provided by the rf parameters obtained from LITRACK. In the ELEGANT tracking, the bunch current peaks at $870 \mathrm{~A}$ in Figs. 6(a) and 6(b). The peak current may be increased to $1 \mathrm{kA}$ by fine-tuning, in which the injector linac phase is increased by $0.15^{\circ}$.

In Fig. 6(c), we also include the geometric wakes of the injector linac and harmonic cavities, calculated for a bunch whose current distribution is frozen between 4 and $215 \mathrm{MeV}$. Using LITRACK to adjust rf parameters by trial and error, we obtained the compressed bunch phase space shown in Fig. 6(c), in which the energy and current are nearly constant for the central portion of the bunch. The wakes of the injector linac and harmonic cavities cause current spikes at the head and tail of the compressed bunch. Figure 6(c) also shows tracking by ELEGANT that includes the injector linac and harmonic cavity geometric wakes, coherent radiation and linac geometric wakes. The ELEGANT tracking confirms that good compression is provided by the rf parameters obtained from LITRACK.

In Figs. 6(a)-6(c), the harmonic cavity voltage was in the range of $8-20 \mathrm{MeV}$, the injector linac phase was between $-21^{\circ}$ and $-18.95^{\circ}$, while the L1 phase was between $-5^{\circ}$ and $13^{\circ}$. A large L2 phase between $40^{\circ}$ 
and $50^{\circ}$ dechirps the bunch before its energy reaches $1.7 \mathrm{GeV}$.

Figure 6 shows that the resistive-wake approximation of CER is useful in compensating wakes by trial and error. By simulating and compensating resistive wakes with a modified version of LITRACK, we obtained the bunch compressor parameters of Table I that can compress a 200-pC parabolic bunch by a factor of 20 to obtain a current of $1 \mathrm{kA}$. Using the resistive-wake approximation of CER may also im- prove the compensation of wakes by reverse tracking of the longitudinal phase space, in comparison with reversetracking that neglects CSR and CER [31].

For the case where coherent radiation and the geometric wakes of the L1 and L2 linacs are included in ELEGANT tracking, Fig. 7 shows details of the compression process. Figure 8 shows details when tracking includes coherent radiation and the geometric wakes of the injector linac, harmonic cavities, L1 and L2 linacs.
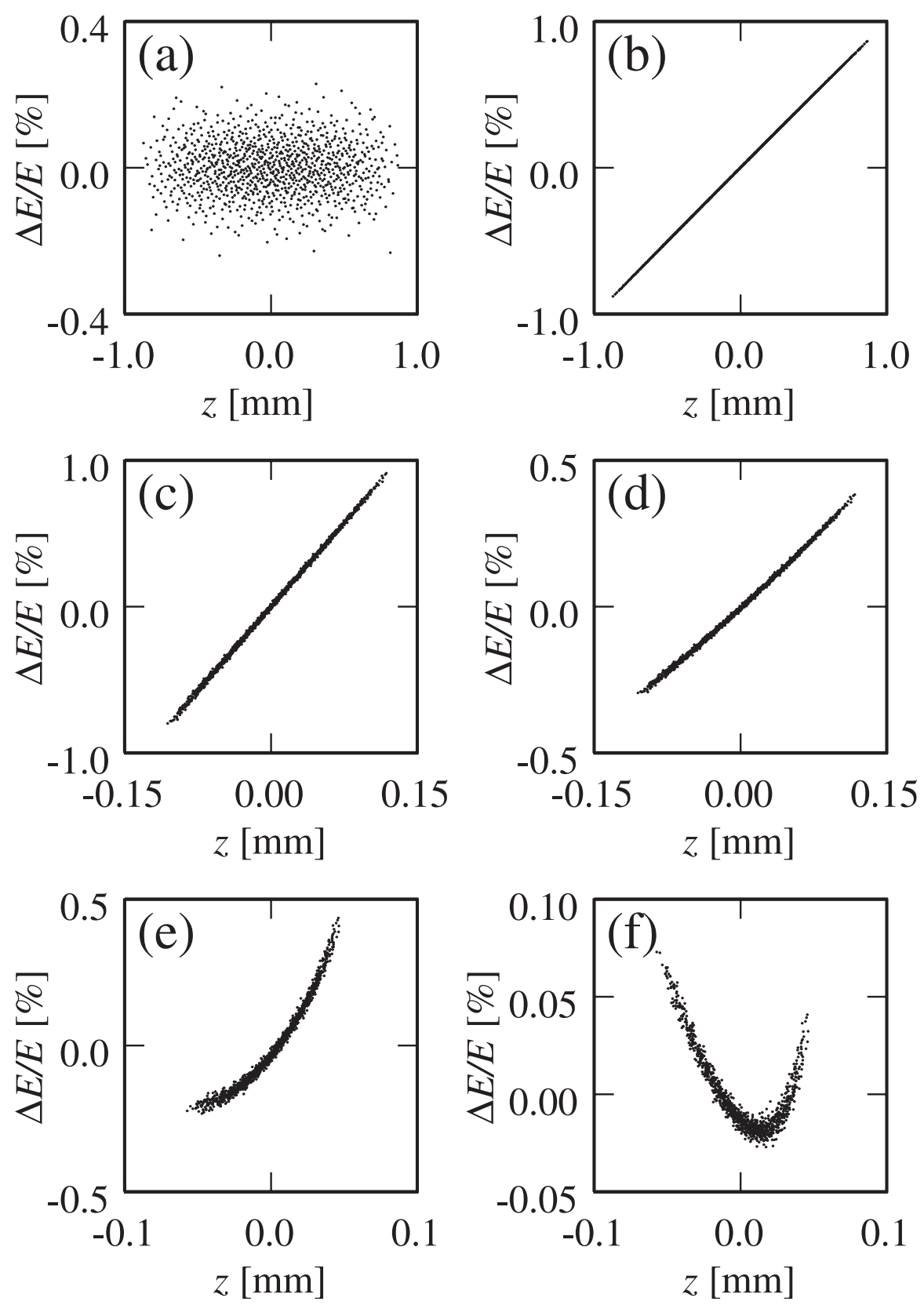

FIG. 7. Bunch compression for an initial parabolic current distribution with rms length of $400 \mu \mathrm{m}$ and charge of $200 \mathrm{pC}$. The longitudinal phase space evolution is given by ELEGANT simulations of 100000 particles that include the coherent-radiation wakes of both chicanes in addition to the geometric wakes of the L1 and L2 linacs. (a) Initial bunch at $4 \mathrm{MeV}$. (b) Chirped bunch at the entrance of the $\mathrm{BC} 1$ chicane. (c) Bunch after single-stage compression in BC1. (d) Chirped bunch at the entrance of the BC2 chicane. (e) Bunch after full compression in BC2. (f) Dechirped bunch at $1.7 \mathrm{GeV}$. 


\section{E. Jitter}

In WiFEL, compressed bunches are seeded with a laser to generate the FEL output. Consequently, the allowable jitter in the bunch arrival time at the FEL with respect to the reference timing is smaller than the compressed bunch duration. Equation (11) may be used to estimate the jitter resulting from variations in current, energy, and chirp at the entrance of the two-stage compressor. We consider the case where $\left|\left(C_{1} R_{56}^{(1)} / E_{1}\right)\left[\Delta E^{\prime}(z)-e R_{0} I^{\prime}(z)\right]\right| \ll 1$, so that upright bunch behavior after $\mathrm{BC} 1$ is safely avoided. In this case, Eq. (11) gives $\partial z_{2}(z) / \partial I(z) \approx\left(-C_{1} C_{2}\right)^{-1}\left[\left(C_{1} R_{56}^{(1)} /\right.\right.$ $\left.\left.E_{1}+C_{1}^{2} C_{2} R_{56}^{(2)} / E_{2}\right) e R_{0}+C_{1}^{2} C_{2} R_{56}^{(2)} e R_{1} / E_{2}\right]$. Thus, the arrival-time jitter $\delta t$ is related to the bunch-to-bunch variation in the initial current profile $\delta I$ by

$$
\begin{aligned}
c \delta t \approx & \mid\left(C_{1} C_{2}\right)^{-1}\left[\left(\frac{C_{1} R_{56}^{(1)}}{E_{1}}+\frac{C_{1}^{2} C_{2} R_{56}^{(2)}}{E_{2}}\right) e R_{0}\right. \\
& \left.+\frac{C_{1}^{2} C_{2} R_{56}^{(2)} e R_{1}}{E_{2}}\right] \mid \delta I .
\end{aligned}
$$
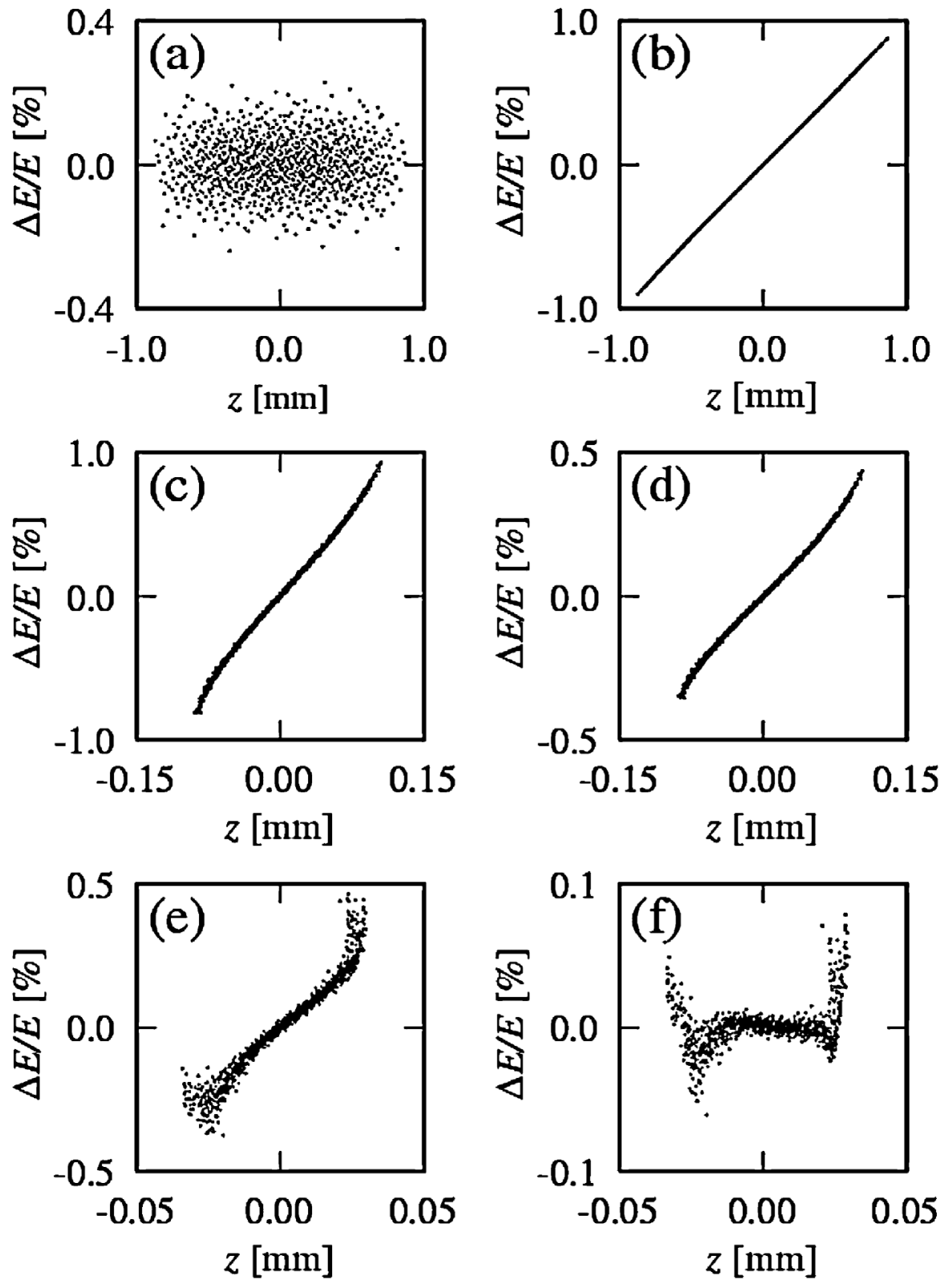

FIG. 8. Bunch compression for an initial parabolic current distribution with rms length of $400 \mu \mathrm{m}$ and charge of $200 \mathrm{pC}$. The longitudinal phase space evolution is given by ELEGANT simulations of 100000 particles that include the coherent-radiation wakes of both chicanes in addition to the geometric wakes of the injector, harmonic cavities, L1 and L2 linacs. (a) Initial bunch at $4 \mathrm{MeV}$. (b) Chirped bunch at the entrance of the BC1 chicane. (c) Bunch after single-stage compression in BC1. (d) Chirped bunch at the entrance of the BC2 chicane. (e) Bunch after full compression in BC2. (f) Dechirped bunch at $1.7 \mathrm{GeV}$. 
From Eq. (11), $\quad \partial z_{2}(z) / \partial \Delta E(z)=\left(C_{1} C_{2}\right)^{-1} \times$ $\left(C_{1} R_{56}^{(1)} / E_{1}+C_{1}^{2} C_{2} R_{56}^{(2)} / E_{2}\right)$, so that the arrival-time jitter is related to the bunch-to-bunch energy variation $\delta E$ at the entrance of $\mathrm{BC} 1$ by

$$
c \delta t=\left|\left(C_{1} C_{2}\right)^{-1}\left(C_{1} R_{56}^{(1)} / E_{1}+C_{1}^{2} C_{2} R_{56}^{(2)} / E_{2}\right)\right| \delta E .
$$

The bunch arrival time is insensitive to $\delta E$ when $C_{1} R_{56}^{(1)} / E_{1}+C_{1}^{2} C_{2} R_{56}^{(2)} / E_{2}=0$, which requires overcompression in one of the stages. [In this case, the total compression is also insensitive to chirp, since $\partial\left(C_{1} C_{2}\right) / \partial h_{1}=$ $-C_{1} C_{2} E_{1}\left(C_{1} R_{56}^{(1)} / E_{1}+C_{1}^{2} C_{2} R_{56}^{(2)} / E_{2}\right)$, where $h_{1}$ is the relative energy chirp.] However, overcompression may cause undesirable large wakes within a chicane magnet, splitting the bunch longitudinally into two bunches [15].

From Eq. (11), $\partial z_{2}(z) / \partial \Delta E^{\prime}(z) \approx C_{1}^{2} R_{56}^{(1)} R_{56}^{(2)} e R_{1} I(z) /$ $E_{1} E_{2}$. For peak current $I_{0}$, the arrival-time jitter is related to the bunch-to-bunch chirp variation $\delta E^{\prime}$ by

$$
c \delta t \approx\left|C_{1}^{2} R_{56}^{(1)} R_{56}^{(2)} e R_{1} I_{0} /\left(E_{1} E_{2}\right)\right| \delta E^{\prime} .
$$

For WiFEL [16], jitter less than $15 \mathrm{fs}$ rms is desired. Evaluating Eqs. (22)-(24) with resistances $R_{0}$ and $R_{1}$ of 327 and $1121 \Omega$, we find that this requires rms errors $\delta I<$ $9.3 \mathrm{~A}, \delta E<9.8 \mathrm{keV}$, and $\delta E^{\prime}<83 \mathrm{MeV} / \mathrm{m}$, in approximate agreement with ELEGANT simulations. The requirement $\delta I<9.3 \mathrm{~A}$ is satisfied if bunches are created at a photocathode with creation-time variation $\ll \sigma_{z} / c=$ $1.3 \mathrm{ps}$ and peak current variation $<9.3 \mathrm{~A}$. Under the assumption of uncorrelated errors in the injector linac cavities, the expected error of $N$ cavities is $N^{1 / 2}$ times the error of a single cavity. For this case, a cavity's amplitude and phase variation must be less than $0.02 \%$ and $0.03^{\circ} \mathrm{rms}$ to achieve $\delta E<9.8 \mathrm{keV}$, in which case the requirement $\delta E^{\prime}<83 \mathrm{MeV} / \mathrm{m}$ is also satisfied. The required amplitude and phase stability are at the current state of the art in control of superconducting cavities [32]. For acceleration from $485 \mathrm{MeV}$ to $1.7 \mathrm{GeV}$ with linac phase of $40^{\circ}-50^{\circ}$, a 15-fs arrival-time jitter after BC2 contributes energy jitter up to $180 \mathrm{keV}$ in the $1.7 \mathrm{GeV}$ bunches, which is smaller than the allowable energy jitter of $\sim 500 \mathrm{keV}$.

\section{MICROBUNCHING}

When a high-current bunch is compressed in magnetic chicanes, longitudinal modulations of the bunch current and energy may grow [2-10]. To prevent large modulations in the compressed bunch, growth may be suppressed by increasing the energy spread of the uncompressed bunch with a laser heater [7,8]. Microbunching growth may be calculated by modeling the effect of CSR, LSC, and/or linac wakes upon the bunch [4-6,8-10]. However, we expect that CER and LSC are the dominant wakes for the WiFEL compressor.

In Appendix B, we model CER, LSC, CSR, and linac geometric wakes with frequency-dependent effective im- pedances, for the case where $Z_{\text {zero }}(k)=0$. At the entrance of the first chicane, we consider a bunch in which each slice has the symmetric normalized energy distribution $f(\delta)$, where $\delta$ is the energy deviation from an ideal linear chirp. This describes an uncompressed bunch with slice energy spread from the electron gun or a matched laser heater. We follow the bunch's evolution through both stages of compression, including the rotated phase space at the entrance of the second chicane $\mathrm{BC} 2$. We obtain analytical formulas for the growth of a small single-wavelength modulation of the bunch current or energy of the form $\Delta I e^{-i \omega_{0} t}$ or $\Delta E e^{-i \omega_{0} t}$. The growth of a sum of small modulations is given by linear superposition.

The frequency-dependent effective impedances downstream of the two chicanes are denoted $Z_{1}(k)$ and $Z_{2}(k)$. For an initial modulation with wave number $k_{0}=\omega_{0} / c$, let $Z_{1} \equiv Z_{1}\left(C_{1} k_{0}\right)$ and $Z_{2} \equiv Z_{2}\left(C_{1} C_{2} k_{0}\right)$.

Each longitudinal slice of the uncompressed bunch (i.e., value of $z$ ) has a distribution of energies and an average energy $\varepsilon(z)$. Similarly, each slice of the compressed bunch has a distribution of energies and an average energy. For a bunch entering $\mathrm{BC} 1$ at energy $E_{1}$, longitudinal modulations of the bunch current $I$ and average energy $E$ obey, for single-stage compression with $Z_{\text {zero }}\left(k_{0}\right)=0$,

$$
\begin{gathered}
T_{I I} \equiv \frac{\Delta I / I_{\text {out }}}{\Delta I / I_{\text {in }}}=F_{1} \\
T_{E I} \equiv \frac{\Delta E / E_{\text {out }}}{\Delta I / I_{\text {in }}}=\frac{-F_{1} C_{1} e Z_{1} I_{0}}{E_{\text {out }}}+\frac{i G_{1} C_{1}}{E_{\text {out }}} \\
T_{I E} \equiv \frac{\Delta I / I_{\text {out }}}{\Delta E / E_{\text {in }}}=i F_{1} C_{1} k_{0} R_{56}^{(1)} \\
T_{E E} \equiv \frac{\Delta E / E_{\text {out }}}{\Delta E / E_{\text {in }}} \\
=\frac{F_{1} C_{1} E_{1}}{E_{\text {out }}}-\frac{G_{1} C_{1}^{2} k_{0} R_{56}^{(1)}}{E_{\text {out }}}-\frac{i F_{1} C_{1}^{2} e Z_{1} I_{0} k_{0} R_{56}^{(1)}}{E_{\text {out }}}
\end{gathered}
$$

where

$$
\begin{aligned}
& F_{1}=\int \cos \left(k_{0} C_{1} R_{56}^{(1)} \delta / E_{1}\right) f(\delta) d \delta, \\
& G_{1}=\int \delta \sin \left(k_{0} C_{1} R_{56}^{(1)} \delta / E_{1}\right) f(\delta) d \delta .
\end{aligned}
$$

The quantities $F_{1}$ and $G_{1}$ describe the suppression of gain at short wavelengths from the bunch's energy spread.

For a Gaussian energy distribution $f(\delta)$, the single-stage gain $(\Delta I / I)_{\text {out }} /(\Delta E / E)_{\text {in }}$ agrees with Eq. 4 of Ref. [4]. Equations (25)-(28) describe the modulation at any location between compression in the $\mathrm{BC} 1$ chicane and the entrance of the BC2 chicane, provided that $E_{\text {out }}$ is the nominal energy at that location and $Z_{1}$ is the effective 
longitudinal impedance experienced after compression in $\mathrm{BC} 1$, prior to reaching that location.

For a bunch entering $\mathrm{BC} 1$ at energy $E_{1}, 2$-stage compression with $Z_{\text {zero }}\left(k_{0}\right)=0$ obeys

$$
\begin{aligned}
T_{I I} \equiv & \frac{\Delta I / I_{\text {out }}}{\Delta I / I_{\text {in }}}=F_{3}-\frac{i F_{1} F_{2} C_{1}^{2} C_{2} e Z_{1} I_{0} k_{0} R_{56}^{(2)}}{E_{2}} \\
T_{E I} \equiv & \frac{\Delta E / E_{\text {out }}}{\Delta I / I_{\text {in }}} \\
= & -\frac{C_{1} C_{2}\left(F_{1} F_{2} Z_{1}+F_{3} Z_{2}\right) e I_{0}}{E_{\text {out }}} \\
& +\frac{F_{1} G_{2} C_{1}^{3} C_{2}^{2} e Z_{1} I_{0} k_{0} R_{56}^{(2)}}{E_{2} E_{\text {out }}} \\
& +\frac{i F_{1} F_{2} C_{1}^{3} C_{2}^{2} e^{2} Z_{1} Z_{2} I_{0}^{2} k_{0} R_{56}^{(2)}}{E_{2} E_{\text {out }}}+\frac{i G_{3} C_{1} C_{2}}{E_{\text {out }}} \\
T_{I E} \equiv & \frac{\Delta I / I_{\text {out }}}{\Delta E / E_{\text {in }}} \\
= & \frac{F_{1} F_{2} C_{1}^{3} C_{2} e Z_{1} I_{0} k_{0}^{2} R_{56}^{(1)} R_{56}^{(2)}}{E_{2}}+i F_{3} C_{1} k_{0} R_{56}^{(1)} \\
& +\frac{i F_{3} C_{1}^{2} C_{2} k_{0} R_{56}^{(2)} E_{1}}{E_{2}}
\end{aligned}
$$

$$
\begin{aligned}
T_{E E} \equiv & \frac{\Delta E / E_{\text {out }}}{\Delta E / E_{\text {in }}} \\
= & \frac{F_{3} C_{1} C_{2} E_{1}}{E_{\text {out }}}-\frac{F_{1} F_{2} C_{1}^{4} C_{2}^{2} e^{2} Z_{1} Z_{2} I_{0}^{2} k_{0}^{2} R_{56}^{(1)} R_{56}^{(2)}}{E_{2} E_{\text {out }}} \\
& -\frac{G_{3} C_{1}^{2} C_{2} k_{0} R_{56}^{(1)}}{E_{\text {out }}}-\frac{G_{3} C_{1}^{3} C_{2}^{2} k_{0} R_{56}^{(2)} E_{1}}{E_{2} E_{\text {out }}} \\
& -\frac{i F_{1} F_{2} C_{1}^{2} C_{2} e Z_{1} I_{0} k_{0} R_{56}^{(1)}}{E_{\text {out }}}-\frac{i F_{3} C_{1}^{2} C_{2} e Z_{2} I_{0} k_{0} R_{56}^{(1)}}{E_{\text {out }}} \\
& -\frac{i F_{3} C_{1}^{3} C_{2}^{2} e Z_{2} I_{0} k_{0} R_{56}^{(2)} E_{1}}{E_{2} E_{\text {out }}} \\
& +\frac{i F_{1} G_{2} C_{1}^{4} C_{2}^{2} e Z_{1} I_{0} k_{0}^{2} R_{56}^{(1)} R_{56}^{(2)}}{E_{2} E_{\text {out }}}
\end{aligned}
$$

where

$$
\begin{aligned}
F_{2} & =\int \cos \left(k_{0} C_{1}^{2} C_{2} R_{56}^{(2)} \delta / E_{2}\right) f(\delta) d \delta, \\
G_{2} & =\int \delta \sin \left(k_{0} C_{1}^{2} C_{2} R_{56}^{(2)} \delta / E_{2}\right) f(\delta) d \delta,
\end{aligned}
$$

and

$$
\begin{aligned}
& F_{3}=\int \cos \left[k_{0}\left(C_{1} R_{56}^{(1)} / E_{1}+C_{1}^{2} C_{2} R_{56}^{(2)} / E_{2}\right) \delta\right] f(\delta) d \delta, \\
& G_{3}=\int \delta \sin \left[k_{0}\left(C_{1} R_{56}^{(1)} / E_{1}+C_{1}^{2} C_{2} R_{56}^{(2)} / E_{2}\right) \delta\right] f(\delta) d \delta .
\end{aligned}
$$

Equations (30)-(33) describe the modulation at any location after compression in the $\mathrm{BC} 2$ chicane, provided that $E_{\text {out }}$ is the nominal energy at that location and $Z_{2}$ is the effective longitudinal impedance experienced by the bunch after compression in $\mathrm{BC} 2$, prior to reaching that location. Since the phase space entering $\mathrm{BC} 2$ is rotated by the previous compression in $\mathrm{BC} 1$, the microbunching growth in $\mathrm{BC} 2$ differs from that given by substituting the $\mathrm{BC} 2$ parameters into Eqs. (25)-(29).

Now consider effective impedance $Z_{\text {zero }}(k)$ upstream of $\mathrm{BC} 1$. In this case, a current modulation $\Delta I e^{-i \omega_{0} t}$ entering $\mathrm{BC} 1$ is accompanied by an energy modulation $-e Z_{\text {zero }}\left(k_{0}\right) \Delta I e^{-i \omega_{0} t}$ after traversing the impedance $Z_{\text {zero }}(k)$. The growth of each modulation is given by Eqs. (25)-(28) or Eqs. (30)-(33), so we easily obtain gain formulas which include the effect of $Z_{\text {zero }}(k)$ :

$$
\frac{\Delta I / I_{\mathrm{out}}}{\Delta I / I_{\mathrm{in}}}=T_{I I}-\frac{e Z_{\mathrm{zero}}\left(k_{0}\right) I_{0}}{E_{1}} T_{I E}
$$

$$
\begin{gathered}
\frac{\Delta E / E_{\text {out }}}{\Delta I / I_{\text {in }}}=T_{E I}-\frac{e Z_{\text {zero }}\left(k_{0}\right) I_{0}}{E_{1}} T_{E E} \\
\frac{\Delta I / I_{\text {out }}}{\Delta E / E_{\text {in }}}=\frac{E_{\text {in }}}{E_{1}} T_{I E} \\
\frac{\Delta E / E_{\text {out }}}{\Delta E / E_{\text {in }}}=\frac{E_{\text {in }}}{E_{1}} T_{E E .} .
\end{gathered}
$$

For single-stage compression, $T_{I I}, T_{I E}, T_{E I}$, and $T_{E E}$ are given by Eqs. (25)-(28), while Eqs. (30)-(33) describe two-stage compression. Equations (36)-(39) may be used to calculate microbunching growth for a bunch with initial energy $E_{\text {in }} \leq E_{1}$, provided that the bunch is effectively frozen at energies between $E_{\text {in }}$ and $E_{1}$. In this case, $Z_{\text {zero }}\left(k_{0}\right)$ describes the total impedance between $E_{\text {in }}$ and $\mathrm{BC}$, including the effective impedances of the first two magnets of $\mathrm{BC} 1$.

For an initial Gaussian energy distribution $f(\delta)$ with rms energy spread of $\sigma_{E}$ [33], the growth suppression from the bunch's energy spread is characterized by 


$$
\begin{aligned}
F_{1}= & \exp \left[-\left(k_{0} C_{1} R_{56}^{(1)} \sigma_{E} / E_{1}\right)^{2} / 2\right], \\
F_{2}= & \exp \left[-\left(k_{0} C_{1}^{2} C_{2} R_{56}^{(2)} \sigma_{E} / E_{2}\right)^{2} / 2\right], \\
F_{3}= & \exp \left\{-\left[k_{0}\left(C_{1} R_{56}^{(1)} / E_{1}+C_{1}^{2} C_{2} R_{56}^{(2)} / E_{2}\right) \sigma_{E}\right]^{2} / 2\right\}, \\
G_{1}= & \left(k_{0} C_{1} R_{56}^{(1)} / E_{1}\right) \sigma_{E}^{2} \exp \left[-\left(k_{0} C_{1} R_{56}^{(1)} \sigma_{E} / E_{1}\right)^{2} / 2\right], \\
G_{2}= & \left(k_{0} C_{1}^{2} C_{2} R_{56}^{(2)} / E_{2}\right) \sigma_{E}^{2} \exp \left[-\left(k_{0} C_{1}^{2} C_{2} R_{56}^{(2)} \sigma_{E} / E_{2}\right)^{2} / 2\right], \\
G_{3}= & k_{0}\left(C_{1} R_{56}^{(1)} / E_{1}+C_{1}^{2} C_{2} R_{56}^{(2)} / E_{2}\right) \sigma_{E}^{2} \\
& \times \exp \left\{-\left[k_{0}\left(C_{1} R_{56}^{(1)} / E_{1}+C_{1}^{2} C_{2} R_{56}^{(2)} / E_{2}\right) \sigma_{E}\right]^{2} / 2\right\} .
\end{aligned}
$$

The curves in Fig. 9(a) show the microbunching gain of the WiFEL 2-stage compressor for a bunch with current of $50 \mathrm{~A}$, normalized transverse emittances of $1 \mu \mathrm{m}-\mathrm{rad}$, and an initial Gaussian energy distribution with $\sigma_{E}=3 \mathrm{keV}$, calculated for the impedances of Fig. 3. At an initial wavelength of $100 \mu \mathrm{m}$, the bunch's emittance reduces the calculated gain by $27 \%$ for an initial energy modulation and by $87 \%$ for an initial current modulation. For the wavelengths shown, the impedance $Z_{\text {zero }}$ is suppressed by the bunch's emittance, so that similar gain curves are obtained if the impedance $Z_{\text {zero }}$ is neglected.

When the initial energy spread results from a laser heater that is matched to the transverse beam size, the distribution $f(\delta)$ is approximately semicircular [8]:

$$
f(\delta)=\left(2 / \pi \delta_{\max }\right) \sqrt{1-\left(\delta / \delta_{\max }\right)^{2}},
$$

where [34] $\delta_{\max }=2 \sigma_{E}$. In this case [35],

$$
\begin{aligned}
F_{1}= & \left(k_{0} C_{1} R_{56}^{(1)} \sigma_{E} / E_{1}\right)^{-1} J_{1}\left(2 k_{0} C_{1} R_{56}^{(1)} \sigma_{E} / E_{1}\right), \\
F_{2}= & \left(k_{0} C_{1}^{2} C_{2} R_{56}^{(2)} \sigma_{E} / E_{2}\right)^{-1} J_{1}\left(2 k_{0} C_{1}^{2} C_{2} R_{56}^{(2)} \sigma_{E} / E_{2}\right), \\
F_{3}= & {\left[k_{0}\left(C_{1} R_{56}^{(1)} / E_{1}+C_{1}^{2} C_{2} R_{56}^{(2)} / E_{2}\right) \sigma_{E}\right]^{-1} } \\
& \times J_{1}\left[2 k_{0}\left(C_{1} R_{56}^{(1)} / E_{1}+C_{1}^{2} C_{2} R_{56}^{(2)} / E_{2}\right) \sigma_{E}\right], \\
G_{1}= & \left(k_{0} C_{1} R_{56}^{(1)} / 2 E_{1}\right)^{-1} J_{2}\left(2 k_{0} C_{1} R_{56}^{(1)} \sigma_{E} / E_{1}\right), \\
G_{2}= & \left(k_{0} C_{1}^{2} C_{2} R_{56}^{(2)} / 2 E_{2}\right)^{-1} J_{2}\left(2 k_{0} C_{1}^{2} C_{2} R_{56}^{(2)} \sigma_{E} / E_{2}\right), \\
G_{3}= & {\left[k_{0}\left(C_{1} R_{56}^{(1)} / E_{1}+C_{1}^{2} C_{2} R_{56}^{(2)} / E_{2}\right) / 2\right]^{-1} } \\
& \times J_{2}\left[2 k_{0}\left(C_{1} R_{56}^{(1)} / E_{1}+C_{1}^{2} C_{2} R_{56}^{(2)} / E_{2}\right) \sigma_{E}\right] .
\end{aligned}
$$

The curves in Fig. 9(b) show the microbunching gain for a semicircular energy distribution with $\sigma_{E}=10 \mathrm{keV}$, calculated for the impedances of Fig. 3. The zeroes of the Bessel functions in Eq. (42) result in localized dips in the gain curve.

To test the gain formulas, we performed ELEGANT tracking of an initial trapezoidal bunch with flattop of $2 \mathrm{~mm}$, head and tail of length $1 \mathrm{~mm}$, and peak current of $50 \mathrm{~A}$. An initial current or energy modulation was applied to the flattop of the bunch, which was then tracked from the entrance of the $\mathrm{BC} 1$ chicane at $215 \mathrm{MeV}$ to the exit of
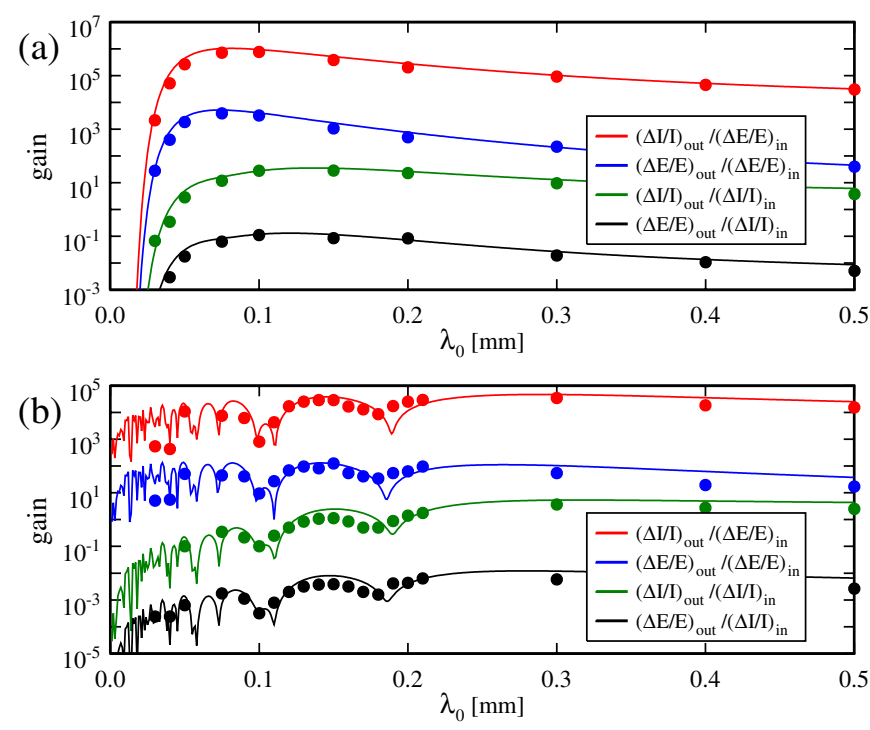

FIG. 9. (Color) Curves show the analytical microbunching gain between the entrance of the $\mathrm{BC} 1$ chicane at $215 \mathrm{MeV}$ and the exit of the L2 linac at $1.7 \mathrm{GeV}$, for a bunch with initial current of $50 \mathrm{~A}$. The gain is shown as a function of the modulation's initial wavelength $\lambda_{0}$. Plotted points show the gain in 4-million particle simulations of a 500-pC bunch with an initial trapezoidal longitudinal profile with flattop length of $2 \mathrm{~mm}$ and flattop current of 50 A. (a) Gaussian energy distribution with initial rms energy spread of $3 \mathrm{keV}$. (b) Semicircular energy distribution from a matched laser heater, with initial rms energy spread of $10 \mathrm{keV}$.

the $\mathrm{L} 2$ linac at $1.7 \mathrm{GeV}$. The tracking includes longitudinal coherent-radiation wakes (i.e., CSR and CER), the longitudinal LSC wakes of the L1 and L2 linacs, in addition to the longitudinal and transverse geometric wakes of the linacs. Noise in the simulations was reduced by tracking 4 million particles whose distributions are populated in each of the six dimensions of phase space by using Halton sequences $[7,29]$. High-frequency noise was further reduced by cutting off the CSR, CER, and LSC wakes at frequencies exceeding the third harmonic of the modulation. To avoid saturation effects, the current modulations in the simulations are $\leq 10 \%$, while the initial energy modulations are smaller than the rms energy spread [4]. For simulations in which the output modulations exceeded numerical noise, the peak-to-peak current variations were measured from histograms of the bunch current and the peak-to-peak energy variations were measured from plots of the longitudinal phase space. The gain in simulations is shown as points in Fig. 9.

The analytic predictions agree with the simulations. For the case of a 10-keV laser heater, we suspect that the wavelengths with localized dips in the gain curve are affected by unequal compression of the bunch, which results in a 5\% variation of the compressed bunch's flattop current.

We also tested the gain formulas for a trapezoidal bunch with normalized transverse emittances of $0.1 \mu \mathrm{m}$-rad and 

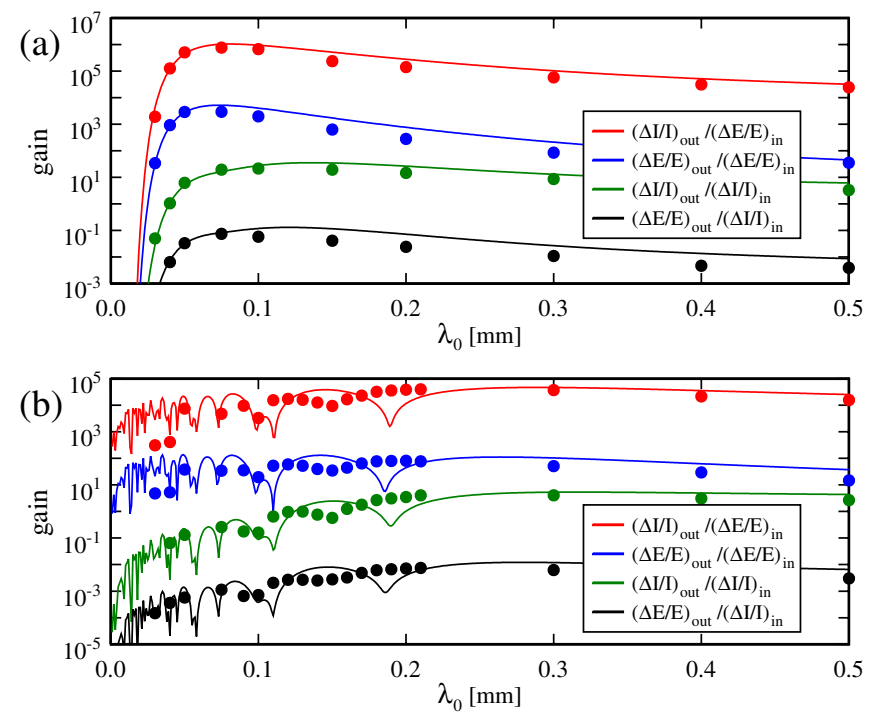

FIG. 10. (Color) Curves show the analytical microbunching gain between the entrance of the $\mathrm{BC} 1$ chicane at $215 \mathrm{MeV}$ and the exit of the L2 linac at $1.7 \mathrm{GeV}$, for a bunch with initial current of $50 \mathrm{~A}$. The gain is shown as a function of the modulation's initial wavelength $\lambda_{0}$. Plotted points show the gain in 4-million particle simulations of a 200-pC parabolic bunch with initial rms bunch length of $400 \mu \mathrm{m}$ and peak current of $50 \mathrm{~A}$. (a) Gaussian energy distribution with initial rms energy spread of $3 \mathrm{keV}$. (b) Semicircular energy distribution from a matched laser heater, with initial rms energy spread of $10 \mathrm{keV}$.

rms Gaussian energy spread of $3 \mathrm{keV}$. The simulations were again in agreement with the formulas.

For a parabolic bunch whose wakes are compensated [as in Fig. 6(b)], we expect that the microbunching gain formulas will apply for wavelengths much shorter than the rms bunch length. For a 200-pC bunch with initial peak current of $50 \mathrm{~A}$, rms length of $400 \mu \mathrm{m}$, and normalized transverse emittances of $1 \mu \mathrm{m}$-rad, Fig. 10 compares the analytic microbunching gain with that observed in ELEGANT simulations, showing approximate agreement even for wavelengths as long as the bunch length. Since a parabolic bunch differs from the constant-current bunch modeled by the gain formulas, the agreement between the formulas and simulations is not as good as in Fig. 9.

When a parabolic bunch with a 3-keV Gaussian energy distribution is heated by $10 \mathrm{keV}$ in a matched laser heater upstream of $\mathrm{BC} 1$, the bunch's energy distribution differs from the semicircular distribution of Eq. (41), which applies for a laser heater acting upon a cold bunch. Figure 11 shows the energy distribution of a parabolic bunch before and after a $10-\mathrm{keV}$ matched laser heater according to an ELEGANT simulation; the heated distribution appears to be a semicircular distribution with Gaussian tails. Figure 12 compares the microbunching gain when the laser heater is simulated by ELEGANT with that predicted analytically for a $10-\mathrm{keV}$ semicircular laser-heater distribution. Approximate agreement is obtained. In this case, the parabolic bunch current distribution differs from the constant-current bunch of the analytic model, and the heated energy distribution differs from the semicircular distribution considered in the analytic model. Nonetheless, reasonable agreement between the formulas and simulations is obtained.

For WiFEL, we require the current and average-energy modulations of the $1.7-\mathrm{GeV}$ compressed bunch to be less than $10 \%$ and $500 \mathrm{keV}$ at wavelengths shorter than the bunch length. Since our analytic gain formulas agree with simulations, we use them to calculate the allowable modulations on the bunch entering the compressor at $215 \mathrm{MeV}$. For a bunch with a $3-\mathrm{keV}$ rms Gaussian energy distribution, the bunch entering the $\mathrm{BC} 1$ chicane must have current modulations less than $0.1 \mathrm{~A}$ and energy modulations less than $12 \mathrm{eV}$. For a bunch with a 10-keV rms energy distribution from a laser heater, the bunch entering $\mathrm{BC} 1$ must have current modulations less than $1 \mathrm{~A}$ and energy modulations less than $470 \mathrm{eV}$.

When a bunch is produced on a photocathode by a laser pulse whose duration equals that of the bunch, laser intensity variations may cause unacceptable modulations. To
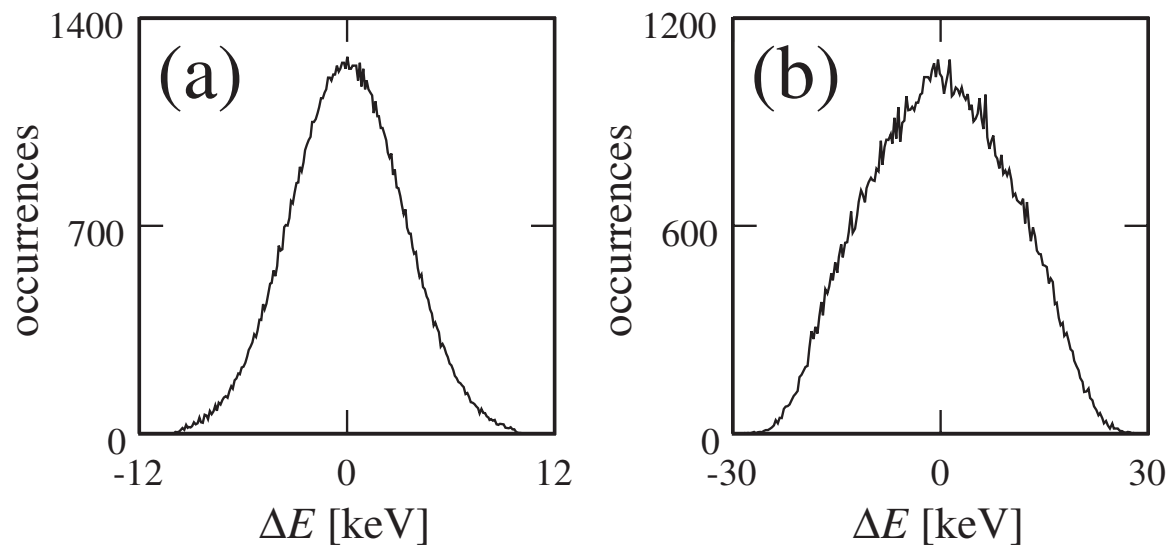

FIG. 11. (a) Gaussian energy distribution with rms energy spread of $3 \mathrm{keV}$. (b) Energy distribution when a 3-keV Gaussian distribution is heated by $10 \mathrm{keV}$ in a laser heater matched to the beam's transverse size. 


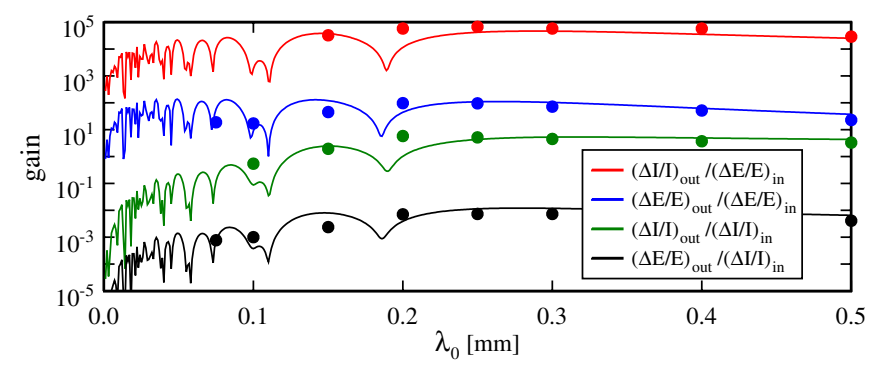

FIG. 12. (Color) Curves show the analytical microbunching gain between the entrance of the $\mathrm{BC} 1$ chicane at $215 \mathrm{MeV}$ and the exit of the L2 linac at $1.7 \mathrm{GeV}$, for a bunch with initial current of $50 \mathrm{~A}$. The gain is calculated as a function of the modulation's initial wavelength $\lambda_{0}$, for a semicircular energy distribution from a matched laser heater with initial rms energy spread of $10 \mathrm{keV}$. Plotted points show the gain in ELEGANT simulations of 13 million particles, for a $200-\mathrm{pC}$ bunch with a parabolic longitudinal profile with initial rms bunch length of $400 \mu \mathrm{m}$ and peak current of $50 \mathrm{~A}$. In the simulations, a bunch with an initial 3-keV Gaussian energy distribution is heated by $10 \mathrm{keV}$ in an ELEGANT simulation of a matched laser heater.

achieve low levels of modulation, we plan to use a laser pulse much shorter than the bunch duration to create a parabolic bunch profile with charge of $200 \mathrm{pC}$, peak current of $50 \mathrm{~A}$, and rms bunch length of $400 \mu \mathrm{m}$. According to Figs. $6-8$, the WiFEL preliminary bunch compressor design can compress such a bunch.

\section{SUMMARY}

For a high-current bunch, wakefields affect the performance of a two-stage compression and acceleration system, where each stage compresses a chirped bunch in a magnetic chicane. The bunch may be affected by the wakes of coherent radiation from the chicane magnets, approximated as coherent steady-state synchrotron radiation (CSR) within the magnets and coherent edge radiation (CER) downstream of the magnets, in addition to the linac geometric and longitudinal space charge (LSC) wakes. An analytic model was obtained which represents the impedances within each chicane by effective impedances before and after the chicane. We applied our model to a preliminary bunch compressor design for the Wisconsin Free Electron Laser (WiFEL).

For macroscopic wavelengths, impedance calculations predict that the WiFEL wakes are dominated by the resistive impedance of CER. By approximating the effective impedances as resistances, we modeled the macroscopic effects. Tracking studies with resistive impedances confirm that this simple model approximates the macroscopic behavior of the WiFEL compressor.

With the resistive-impedance approximation, we calculated the minimum initial bunch length that can be compressed without producing an upright tail in phase space. We used resistive impedances to compensate wakes by adjusting rf parameters. We also obtained approximate formulas for the jitter in the bunch arrival time caused by bunch-to-bunch variation in current, energy, and chirp.

Formulas for microbunching gain were derived for an initial small single-wavelength modulation, affected by frequency-dependent effective impedances before and after each chicane. For a given current or energy modulation at the entrance of the two-stage compressor, the formulas predict the output current and energy modulations, including the gain suppression at short wavelengths from the bunch's energy spread and emittance. The gain was calculated for a 3-keV rms Gaussian energy spread and for a 10-keV rms energy distribution produced by a matched laser heater. Tracking studies verify the approximate validity of the gain formulas.

For the preliminary WiFEL bunch compressor, we calculated the minimum compressible initial bunch length, the jitter from bunch-to-bunch variations in current, energy, and chirp, and the microbunching growth. We plan to compress a 200-pC bunch with peak current of $50 \mathrm{~A}$ and rms bunch length of $400 \mu \mathrm{m}$ that is produced by illuminating a photocathode with a laser pulse much shorter than the temporal bunch length. With state-of-the-art control of the linac's superconducting cavities, we expect to obtain acceptable performance as we compress the bunch by a factor of 20, obtaining a peak current of $1 \mathrm{kA}$.

\section{ACKNOWLEDGMENTS}

The authors thank P. Emma, M. Borland, W. S. Graves, R. A. Legg, J. J. Bisognano, M. A. Green, and K. D. Jacobs for useful suggestions. This work of R. A. Bosch and K. J. Kleman was conducted at the Synchrotron Radiation Center, which is supported by National Science Foundation Award No. DMR-0537588 and the University of Wisconsin-Madison. The work of J. Wu was supported by the U.S. Department of Energy under Contract No. DEAC02-76SF00515, and the work was performed in support of the Linac Coherent Light Source project at the Stanford Linear Accelerator Center.

\section{APPENDIX A: RESISTIVE IMPEDANCE}

Consider a cold, linearly chirped electron bunch that is compressed in the $\mathrm{BC} 1$ and $\mathrm{BC} 2$ chicanes. At the entrance of the first chicane $\mathrm{BC} 1$, let $z=c t$ parametrize the bunch (where $t$ is the electron arrival time) with $z=0$ for the reference particle and $z>0$ at the tail, where $c$ is the speed of light. Let $\varepsilon(z)$ be the energy profile, $I(z)>0$ the bunch current magnitude, while the electron charge magnitude is $e>0$.

In the absence of collective effects, $\mathrm{BC} 1$ compresses at energy $E_{1}$, where an incoming bunch has linear energy chirp $d \varepsilon / d z=h_{1} E_{1}$, with $h_{1} \equiv(d \varepsilon / d z) / E_{1}$. After the bunch exits $\mathrm{BC} 1$, let $z_{1}$ parametrize the bunch. The bunch compression is characterized by the transfer matrix ele- 
ment $R_{56}^{(1)} \equiv \partial z_{1} / \partial\left\{\left[\varepsilon(z)-E_{1}\right] / E_{1}\right\}<0$. The compression ratio $C_{1}$ obeys $C_{1}^{-1}=1+h_{1} R_{56}^{(1)}$, where $C_{1}>0$ for undercompression and $C_{1}<0$ for overcompression (in which the head and tail of the bunch are exchanged). For compression in a chicane, $R_{56}^{(1)}$ is negative and $h_{1}$ is positive in our notation. The energy profile after $\mathrm{BC} 1$ is $\varepsilon_{1}\left(z_{1}\right)$.

The linac $\mathrm{L} 1$ between $\mathrm{BC} 1$ and $\mathrm{BC} 2$ imparts energy $E_{2}-E_{1}$ and additional chirp $d \varepsilon / d z_{1}=h_{2} E_{2}$, and the energy profile becomes $\varepsilon_{L}\left(z_{1}\right)$.

The chicane $\mathrm{BC} 2$ compresses at energy $E_{2}$. After the bunch exits $\mathrm{BC} 2$, let $z_{2}$ parametrize the bunch. The bunch compression is characterized by the transfer matrix element $R_{56}^{(2)} \equiv \partial z_{2} / \partial\left\{\left[\varepsilon_{L}\left(z_{1}\right)-E_{2}\right] / E_{2}\right\}<0$. The compression ratio $C_{2}$ obeys $C_{2}^{-1}=1+R_{56}^{(2)}\left(h_{2}+C_{1} h_{1} E_{1} / E_{2}\right)$.

The linac L2 imparts energy $E_{f}-E_{2}$ and additional chirp $d \varepsilon / d z_{2}=h_{f} E_{f}$, which may be chosen to obtain a dechirped compressed bunch at the final energy $E_{f}$.

To approximate the macroscopic effect of longitudinal wakes for a bunch with a nonzero current, we consider resistive impedance $R_{0}$ before the $\mathrm{BC} 1$ chicane, resistive impedance $R_{1}$ between the $\mathrm{BC} 1$ and $\mathrm{BC} 2$ chicanes, and resistive impedance $R_{2}$ after the $\mathrm{BC} 2$ chicane. We use the notation $T_{1} \equiv R_{56}^{(1)} / E_{1}, T_{2} \equiv R_{56}^{(2)} / E_{2}, S_{1} \equiv h_{1} E_{1}, S_{2} \equiv$ $h_{2} E_{2}$, and $S_{f} \equiv h_{f} E_{f}$.

Consider a bunch current $I(z)$ at the entrance of the bunch compressor, where the bunch has energy profile

$$
\varepsilon_{\text {in }}(z)=E_{1}+S_{1} z+\Delta E(z)
$$

Here, $\Delta E(z)$ is the difference between the bunch energy profile and an ideal linear chirp. After passing through the effective resistance $R_{0}$, the energy profile becomes

$$
\varepsilon(z)=E_{1}+S_{1} z+\Delta E(z)-e R_{0} I(z) .
$$

The compression in $\mathrm{BC} 1$ obeys

$$
\begin{aligned}
z_{1}(z) & =z+\left(R_{56}^{(1)} / E_{1}\right)\left[\varepsilon(z)-E_{1}\right] \\
& =z / C_{1}+T_{1}\left[\Delta E(z)-e R_{0} I(z)\right],
\end{aligned}
$$

with derivative with respect to $z$ of

$$
z_{1}^{\prime}(z)=C_{1}^{-1}\left\{1+C_{1} T_{1}\left[\Delta E^{\prime}(z)-e R_{0} I^{\prime}(z)\right]\right\} .
$$

When the rhs of Eq. (A4) is zero, an upright bunch occurs after $\mathrm{BC} 1$. Thus, part of the bunch is upright after the first compressor when

$$
\Delta E^{\prime}(z)-e R_{0} I^{\prime}(z)=-1 / C_{1} T_{1}=-E_{1} / C_{1} R_{56}^{(1)} .
$$

The function $z_{1}(z)$ is invertible if its derivative is nonzero for all values of $z$, with inverse $z\left(z_{1}\right)$. After compression in $\mathrm{BC} 1$, the current profile becomes

$$
I_{1}\left(z_{1}\right)=\frac{I\left[z\left(z_{1}\right)\right]}{d z_{1} / d z}=\frac{C_{1} I\left[z\left(z_{1}\right)\right]}{1+C_{1} T_{1}\left\{\Delta E^{\prime}\left[z\left(z_{1}\right)\right]-e R_{0} I^{\prime}\left[z\left(z_{1}\right)\right]\right\}} .
$$

After passing through the resistive wake and linac downstream of $\mathrm{BC} 1$, the energy profile becomes

$$
\begin{aligned}
\varepsilon_{L}\left(z_{1}\right)= & \varepsilon\left[z\left(z_{1}\right)\right]-e R_{1} I_{1}\left(z_{1}\right)+E_{2}-E_{1}+S_{2} z_{1} \\
= & E_{2}+\left(S_{1}+\frac{S_{2}}{C_{1}}\right) z\left(z_{1}\right) \\
& +\left(1+S_{2} T_{1}\right)\left\{\Delta E\left[z\left(z_{1}\right)\right]-e R_{0} I\left[z\left(z_{1}\right)\right]\right\} \\
& -\frac{e R_{1} C_{1} I\left[z\left(z_{1}\right)\right]}{1+C_{1} T_{1}\left\{\Delta E^{\prime}\left[z\left(z_{1}\right)\right]-e R_{0} I^{\prime}\left[z\left(z_{1}\right)\right]\right\}} .
\end{aligned}
$$

Compression in BC2 obeys $z_{2}\left(z_{1}\right)=z_{1}+\left(R_{56}^{(2)} / E_{2}\right) \times$ $\left[\varepsilon_{L}\left(z_{1}\right)-E_{2}\right]=z_{1}+T_{2}\left[\varepsilon_{L}\left(z_{1}\right)-E_{2}\right]$, so that

$$
\begin{aligned}
z_{2}(z)= & \frac{1}{C_{1} C_{2}}\left\{z+\left(C_{1} T_{1}+C_{1}^{2} C_{2} T_{2}\right)\left[\Delta E(z)-e R_{0} I(z)\right]\right. \\
& \left.-\frac{C_{1}^{2} C_{2} T_{2} e R_{1} I(z)}{1+C_{1} T_{1}\left[\Delta E^{\prime}(z)-e R_{0} I^{\prime}(z)\right]}\right\}
\end{aligned}
$$

with derivative

$$
\begin{aligned}
\frac{d z_{2}}{d z}= & \frac{1}{C_{1} C_{2}}\left\{1+\left(C_{1} T_{1}+C_{1}^{2} C_{2} T_{2}\right)\left[\Delta E^{\prime}(z)-e R_{0} I^{\prime}(z)\right]\right. \\
& -\frac{C_{1}^{2} C_{2} T_{2} e R_{1} I^{\prime}(z)}{1+C_{1} T_{1}\left[\Delta E^{\prime}(z)-e R_{0} I^{\prime}(z)\right]} \\
& \left.+\frac{C_{1}^{3} C_{2} T_{1} T_{2} e R_{1} I(z)\left[\Delta E^{\prime \prime}(z)-e R_{0} I^{\prime \prime}(z)\right]}{\left\{1+C_{1} T_{1}\left[\Delta E^{\prime}(z)-e R_{0} I^{\prime}(z)\right]\right\}^{2}}\right\}
\end{aligned}
$$

When the rhs of Eq. (A9) is zero, an upright bunch occurs after $\mathrm{BC} 2$. The function $z_{2}(z)$ is invertible if its derivative is nonzero for all values of $z$, in which case we let $z\left(z_{2}\right)$ denote the inverse. The current profile after $\mathrm{BC} 2$ is

$$
I_{2}\left(z_{2}\right)=\frac{I\left[z\left(z_{2}\right)\right]}{d z_{2} / d z} .
$$

After the resistive impedance $R_{2}$ and acceleration in the L2 linac, the energy profile becomes

$$
\varepsilon_{f}\left(z_{2}\right)=\varepsilon_{L}\left[z_{1}\left(z_{2}\right)\right]-e R_{2} I_{2}\left(z_{2}\right)+E_{f}-E_{2}+S_{f} z_{2} .
$$

\section{APPENDIX B: MICROBUNCHING}

In this Appendix, we consider microbunching from effective impedances $Z_{1}(k)$ and $Z_{2}(k)$ after each stage of compression, without considering the effective impedance $Z_{\text {zero }}(k)$ upstream of the first chicane. To model the gain suppression from the bunch's energy spread, we consider a distribution of electron energies. At the entrance of the first chicane, we assume that each slice of the bunch has the same symmetric normalized energy distribution $f(\delta)$, where $\delta$ is the energy deviation from an ideal linear chirp. This describes an uncompressed bunch whose energy spread results from the electron gun or a matched laser heater. After each chicane, the bunch distribution is rotated in phase space, so we do not make this assumption. 
We use coordinates $(z, \delta)$ for the initial bunch, $\left(z_{1}, \delta\right)$ after the first stage of compression, and $\left(z_{2}, \delta\right)$ for the final bunch after two stages of compression, where $\delta$ is the initial energy deviation from an ideal linear chirp. For small modulations where the compressed bunch is not upright in phase space, these are valid coordinates with invertible transformations. The transformation from $(z, \delta)$ to $\left(z_{1}, \delta\right)$ has Jacobian $\partial z_{1} / \partial z$ while the transformation from $(z, \delta)$ to $\left(z_{2}, \delta\right)$ has Jacobian $\partial z_{2} / \partial z$. We will characterize the suppression of microbunching from energy spread with expressions $F_{1}, F_{2}, F_{3}, G_{1}, G_{2}$, and $G_{3}$, which depend upon the initial energy distribution $f(\delta)$.

For brevity, we use the notation $T_{1} \equiv R_{56}^{(1)} / E_{1}, T_{2} \equiv$ $R_{56}^{(2)} / E_{2}, S_{1} \equiv h_{1} E_{1}, S_{2} \equiv h_{2} E_{2}$, and $S_{f} \equiv h_{f} E_{f}$. We let $\hat{Z}_{1}(k)$ represent the portion of the impedance $Z_{1}(k)$ that is experienced upstream of the L1 linac, where the bunch's nominal energy is $E_{1}$. Similarly, we let $\hat{Z}_{2}(k)$ represent the portion of the impedance $Z_{2}(k)$ that is experienced upstream of the L2 linac, where the bunch's nominal energy is $E_{2}$. For an initial modulation with wave number $k_{0}$, let $R_{1} \equiv Z_{1}(0), \quad Z_{1} \equiv Z_{1}\left(C_{1} k_{0}\right), \quad R_{2} \equiv Z_{2}(0), \quad$ and $\quad Z_{2} \equiv$ $Z_{2}\left(C_{1} C_{2} k_{0}\right)$. Let $\quad \hat{R}_{1} \equiv \hat{Z}_{1}(0), \quad \hat{Z}_{1} \equiv \hat{Z}_{1}\left(C_{1} k_{0}\right), \quad \hat{R}_{2} \equiv$ $\hat{Z}_{2}(0)$, and $\hat{Z}_{2} \equiv \hat{Z}_{2}\left(C_{1} C_{2} k_{0}\right)$.

\section{Current modulation}

Consider an initial current modulation $\Delta I e^{-i \omega_{0} t}=$ $\Delta I e^{-i k_{0} z}$, where $t$ is the arrival time and the coordinate $z$ increases toward the tail of the bunch. The bunch current is

$$
I(z)=I_{0}+\Delta I e^{-i k_{0} z} .
$$

We assume that the current density in phase space at the entrance of $\mathrm{BC} 1$ is $I(z, \delta)=I(z) f(\delta)$, where $I(z)$ is the bunch current. At the entrance of $\mathrm{BC} 1$, let $\varepsilon(z, \delta)$ equal the energy at coordinate $(z, \delta)$, while $\varepsilon(z)$ is the average energy at longitudinal position $z$. We have

$$
\begin{gathered}
\varepsilon(z, \delta)=E_{1}+S_{1} z+\delta \\
\varepsilon(z)=\int \varepsilon(z, \delta) f(\delta) d \delta=E_{1}+S_{1} z .
\end{gathered}
$$

The compression in $\mathrm{BC} 1$ gives the transformation

$$
z_{1}(z, \delta)=z+T_{1}\left[\varepsilon(z, \delta)-E_{1}\right]=z / C_{1}+T_{1} \delta,
$$

with inverse

$$
z\left(z_{1}, \delta\right)=C_{1} z_{1}-C_{1} T_{1} \delta
$$

After $\mathrm{BC} 1$, the current density in coordinates $\left(z_{1}, \delta\right)$ is

$$
\begin{aligned}
I_{1}\left(z_{1}, \delta\right) & =I\left[z\left(z_{1}, \delta\right), \delta\right] /\left(\partial z_{1} / \partial z\right) \\
& =C_{1}\left(I_{0}+\Delta I e^{-i k_{0}\left(C_{1} z_{1}-C_{1} T_{1} \delta\right)}\right) f(\delta) .
\end{aligned}
$$

The current after $\mathrm{BC} 1$ equals

$$
I_{1}\left(z_{1}\right)=\int I_{1}\left(z_{1}, \delta\right) d \delta=C_{1}\left(I_{0}+F_{1} \Delta I e^{-i k_{0} C_{1} z_{1}}\right),
$$

where

$$
F_{1}=\int \cos \left(k_{0} C_{1} T_{1} \delta\right) f(\delta) d \delta .
$$

The bunch passes through the CSR and CER impedance upstream of the L1 linac, $\hat{Z}_{1}(k)$, where $\hat{Z}_{1}(0) \equiv \hat{R}_{1}$ and $\hat{Z}_{1}\left(C_{1} k_{0}\right) \equiv \hat{Z}_{1}$. Using the relation $C_{1}=1-S_{1} C_{1} T_{1}$ gives the energy at the entrance of the L1 linac in coordinates $\left(z_{1}, \delta\right)$

$$
\begin{aligned}
\varepsilon_{1}\left(z_{1}, \delta\right)= & \varepsilon\left[z\left(z_{1}, \delta\right), \delta\right]-(e / 2 \pi) \int d k \hat{Z}_{1}(k) I_{1}(k) e^{-i k z_{1}} \\
= & E_{1}+S_{1} C_{1} z_{1}+C_{1} \delta \\
& -e C_{1}\left(\hat{R}_{1} I_{0}+F_{1} \hat{Z}_{1} \Delta I e^{-i k_{0} C_{1} z_{1}}\right)
\end{aligned}
$$

The average energy of electrons at coordinate $z_{1}, \varepsilon_{1}\left(z_{1}\right)$, obeys $\varepsilon_{1}\left(z_{1}\right) I_{1}\left(z_{1}\right)=\int d \delta \varepsilon_{1}\left(z_{1}, \delta\right) I_{1}\left(z_{1}, \delta\right)$. To first order in $\Delta I$, this gives

$$
\begin{aligned}
\varepsilon_{1}\left(z_{1}\right)= & E_{1}+S_{1} C_{1} z_{1}-e C_{1} \hat{R}_{1} I_{0}-e F_{1} C_{1} \hat{Z}_{1} \Delta I e^{-i k_{0} C_{1} z_{1}} \\
& +i G_{1} C_{1}\left(\Delta I / I_{0}\right) e^{-i k_{0} C_{1} z_{1},}
\end{aligned}
$$

where

$$
G_{1}=\int \delta \sin \left(k_{0} C_{1} T_{1} \delta\right) f(\delta) d \delta .
$$

After passing through the L1 linac, the energy is

$$
\begin{aligned}
\varepsilon_{L}\left(z_{1}, \delta\right)= & E_{2}+\left(S_{1} C_{1}+S_{2}\right) z_{1}+C_{1} \delta \\
& -e C_{1}\left(R_{1} I_{0}+F_{1} Z_{1} \Delta I e^{-i k_{0} C_{1} z_{1}}\right) .
\end{aligned}
$$

To first order in $\Delta I$, the average energy of electrons at coordinate $z_{1}, \varepsilon_{L}\left(z_{1}\right)$, is

$$
\begin{aligned}
\varepsilon_{L}\left(z_{1}\right)= & E_{2}+\left(S_{1} C_{1}+S_{2}\right) z_{1}-e C_{1} R_{1} I_{0} \\
& -e F_{1} C_{1} Z_{1} \Delta I e^{-i k_{0} C_{1} z_{1}} \\
& +i G_{1} C_{1}\left(\Delta I / I_{0}\right) e^{-i k_{0} C_{1} z_{1}} .
\end{aligned}
$$

From Eqs. (B7), (B10), and (B13), the current and averageenergy modulations for single-stage compression obey

$$
\begin{gathered}
T_{I I} \equiv \frac{\Delta I / I_{\text {out }}}{\Delta I / I_{\text {in }}}=F_{1}, \\
T_{E I} \equiv \frac{\Delta E / E_{\text {out }}}{\Delta I / I_{\text {in }}}=\frac{-F_{1} C_{1} e Z_{1} I_{0}}{E_{\text {out }}}+\frac{i G_{1} C_{1}}{E_{\text {out }}} .
\end{gathered}
$$

Equations (B14) and (B15) describe the modulation at any location between compression in $\mathrm{BC} 1$ and the entrance of $\mathrm{BC} 2$, provided that $E_{\text {out }}$ is the nominal bunch energy at that location and $Z_{1}$ is the longitudinal impedance experienced after compression in $\mathrm{BC} 1$, prior to reaching that location. 
The compression in $\mathrm{BC} 2$ gives the transformation

$$
\begin{aligned}
z_{2}\left(z_{1}, \delta\right)= & z_{1}+T_{2}\left[\varepsilon_{L}\left(z_{1}, \delta\right)-E_{2}\right] \\
= & z_{1} / C_{2}+C_{1} T_{2} \delta-C_{1} T_{2} e\left(R_{1} I_{0}\right. \\
& \left.+F_{1} Z_{1} \Delta I e^{-i k_{0} C_{1} z_{1}}\right) .
\end{aligned}
$$

We can relate $z_{2}$ and $z$ by using Eq. (B4),

$$
\begin{aligned}
z_{2}(z, \delta)= & z / C_{1} C_{2}+\left(T_{1} / C_{2}+T_{2} C_{1}\right) \delta \\
& -C_{1} T_{2} e\left(R_{1} I_{0}+F_{1} Z_{1} \Delta I e^{-i k_{0}\left(z+C_{1} T_{1} \delta\right)}\right),
\end{aligned}
$$

$$
\begin{aligned}
\partial z_{2} / \partial z(z, \delta)= & \left(C_{1} C_{2}\right)^{-1} \\
& \times\left(1+i F_{1} C_{1}^{2} C_{2} T_{2} e Z_{1} k_{0} \Delta I e^{-i k_{0}\left(z+C_{1} T_{1} \delta\right)}\right) .
\end{aligned}
$$

When the real part of this derivative equals zero, upright bunch regions occurs after BC2. The energy spread modifies the threshold value of $\Delta I$ by the factor $F_{1}^{-1}$.

The following equations are valid to first order in $\Delta I$. The inverse of Eq. (B16) is

with derivative

$$
z_{1}\left(z_{2}, \delta\right)=C_{2} z_{2}-C_{1} C_{2} T_{2} \delta+C_{1} C_{2} T_{2} e R_{1} I_{0}+F_{1} C_{1} C_{2} T_{2} e Z_{1} \Delta I e^{-i k_{0}\left(C_{1} C_{2} z_{2}+C_{1}^{2} C_{2} T_{2} e R_{1} I_{0}-C_{1}^{2} C_{2} T_{2} \delta\right)} .
$$

The inverse of Eq. (B17) is

$$
z\left(z_{2}, \delta\right)=C_{1} C_{2} z_{2}+C_{1}^{2} C_{2} T_{2} e R_{1} I_{0}-\left(C_{1} T_{1}+C_{1}^{2} C_{2} T_{2}\right) \delta+F_{1} C_{1}^{2} C_{2} T_{2} e Z_{1} \Delta I e^{-i k_{0}\left(C_{1} C_{2} z_{2}+C_{1}^{2} C_{2} T_{2} e R_{1} I_{0}-C_{1}^{2} C_{2} T_{2} \delta\right)} .
$$

The current density after $\mathrm{BC} 2$ in coordinates $\left(z_{2}, \delta\right)$ is

$$
\begin{aligned}
I_{2}\left(z_{2}, \delta\right)= & \frac{I\left[z\left(z_{2}, \delta\right), \delta\right]}{\partial z_{2} / \partial z}=\frac{C_{1} C_{2}\left[I_{0}+\Delta I e^{-i k_{0} z\left(z_{2}, \delta\right)}\right] f(\delta)}{1+i F_{1} C_{1}^{2} C_{2} T_{2} e Z_{1} k_{0} \Delta I e^{-i k_{0}\left[z\left(z_{2}, \delta\right)+C_{1} T_{1} \delta\right]}} \\
= & C_{1} C_{2} f(\delta) I_{0}\left[1+\left(\Delta I / I_{0}\right) e^{-i k_{0}\left[C_{1} C_{2} z_{2}+C_{1}^{2} C_{2} T_{2} e R_{1} I_{0}-\left(C_{1} T_{1}+C_{1}^{2} C_{2} T_{2}\right) \delta\right]}\right. \\
& \left.-i F_{1} C_{1}^{2} C_{2} T_{2} e Z_{1} k_{0} \Delta I e^{-i k_{0}\left(C_{1} C_{2} z_{2}+C_{1}^{2} C_{2} T_{2} e R_{1} I_{0}-C_{1}^{2} C_{2} T_{2} \delta\right)}\right] .
\end{aligned}
$$

The current is

$$
I_{2}\left(z_{2}\right)=\int I_{2}\left(z_{2}, \delta\right) d \delta=C_{1} C_{2} I_{0}\left[1+\left(F_{3} / I_{0}-i F_{1} F_{2} C_{1}^{2} C_{2} T_{2} e Z_{1} k_{0}\right) \Delta I e^{-i k_{0}\left(C_{1} C_{2} z_{2}+C_{1}^{2} C_{2} T_{2} e R_{1} I_{0}\right)}\right]
$$

where

$$
F_{2}=\int \cos \left(k_{0} C_{1}^{2} C_{2} T_{2} \delta\right) f(\delta) d \delta, \quad F_{3}=\int \cos \left[k_{0}\left(C_{1} T_{1}+C_{1}^{2} C_{2} T_{2}\right) \delta\right] f(\delta) d \delta
$$

After passing through the CSR and CER impedance $\hat{Z}_{2}(k)$, where $\hat{Z}_{2}(0) \equiv \hat{R}_{2}$ and $\hat{Z}_{2}\left(C_{1} C_{2} k_{0}\right) \equiv \hat{Z}_{2}$, using the relation $C_{2}=1-\left(S_{1} C_{1}+S_{2}\right) T_{2} C_{2}$ gives the energy in coordinates $\left(z_{2}, \delta\right)$ :

$$
\begin{aligned}
\varepsilon_{2}\left(z_{2}, \delta\right)= & \varepsilon_{L}\left[z_{1}\left(z_{2}, \delta\right), \delta\right]-(e / 2 \pi) \int d k \hat{Z}_{2}(k) I_{2}(k) e^{-i k z_{2}} \\
= & E_{2}-C_{1} C_{2} e R_{1} I_{0}-C_{1} C_{2} e \hat{R}_{2} I_{0}+\left(S_{1} C_{1}+S_{2}\right) C_{2} z_{2}+C_{1} C_{2} \delta \\
& -F_{1} C_{1} C_{2} e Z_{1} \Delta I e^{-i k_{0}\left(C_{1} C_{2} z_{2}+C_{1}^{2} C_{2} T_{2} e R_{1} I_{0}-C_{1}^{2} C_{2} T_{2} \delta\right)}-F_{3} C_{1} C_{2} e \hat{Z}_{2} \Delta I e^{-i k_{0}\left(C_{1} C_{2} z_{2}+C_{1}^{2} C_{2} T_{2} e R_{1} I_{0}\right)} \\
& +i F_{1} F_{2} C_{1}^{3} C_{2}^{2} T_{2} e^{2} Z_{1} \hat{Z}_{2} k_{0} I_{0} \Delta I e^{-i k_{0}\left(C_{1} C_{2} z_{2}+C_{1}^{2} C_{2} T_{2} e R_{1} I_{0}\right)} .
\end{aligned}
$$

The average energy of electrons at coordinate $z_{2}, \varepsilon_{2}\left(z_{2}\right)$, obeys $\varepsilon_{2}\left(z_{2}\right) I_{2}\left(z_{2}\right)=\int d \delta \varepsilon_{2}\left(z_{2}, \delta\right) I_{2}\left(z_{2}, \delta\right)$, so that

$$
\begin{aligned}
\varepsilon_{2}\left(z_{2}\right)= & E_{2}-C_{1} C_{2} e R_{1} I_{0}-C_{1} C_{2} e \hat{R}_{2} I_{0}+\left(S_{1} C_{1}+S_{2}\right) C_{2} z_{2}+\left(-F_{1} F_{2} C_{1} C_{2} e Z_{1}-F_{3} C_{1} C_{2} e \hat{Z}_{2}\right. \\
& \left.+i F_{1} F_{2} C_{1}^{3} C_{2}^{2} T_{2} e^{2} Z_{1} \hat{Z}_{2} k_{0} I_{0}+i G_{3} C_{1} C_{2} / I_{0}+F_{1} G_{2} C_{1}^{3} C_{2}^{2} T_{2} e Z_{1} k_{0}\right) \Delta I e^{-i k_{0}\left(C_{1} C_{2} z_{2}+C_{1}^{2} C_{2} T_{2} e R_{1} I_{0}\right)}
\end{aligned}
$$

where

$$
G_{2}=\int \delta \sin \left(k_{0} C_{1}^{2} C_{2} T_{2} \delta\right) f(\delta) d \delta, \quad G_{3}=\int \delta \sin \left[k_{0}\left(C_{1} T_{1}+C_{1}^{2} C_{2} T_{2}\right) \delta\right] f(\delta) d \delta .
$$

After passing through the L2 linac, the final energy is 


$$
\begin{aligned}
\varepsilon_{f}\left(z_{2}, \delta\right)= & E_{f}-C_{1} C_{2} e R_{1} I_{0}-C_{1} C_{2} e R_{2} I_{0}+\left(S_{1} C_{1} C_{2}+S_{2} C_{2}+S_{f}\right) z_{2}+C_{1} C_{2} \delta \\
& -F_{1} C_{1} C_{2} e Z_{1} \Delta I e^{-i k_{0}\left(C_{1} C_{2} z_{2}+C_{1}^{2} C_{2} T_{2} e R_{1} I_{0}-C_{1}^{2} C_{2} T_{2} \delta\right)}-F_{3} C_{1} C_{2} e Z_{2} \Delta I e^{-i k_{0}\left(C_{1} C_{2} z_{2}+C_{1}^{2} C_{2} T_{2} e R_{1} I_{0}\right)} \\
& +i F_{1} F_{2} C_{1}^{3} C_{2}^{2} T_{2} e^{2} Z_{1} Z_{2} k_{0} I_{0} \Delta I e^{-i k_{0}\left(C_{1} C_{2} z_{2}+C_{1}^{2} C_{2} T_{2} e R_{1} I_{0}\right)} .
\end{aligned}
$$

The average energy at coordinate $z_{2}, \varepsilon_{f}\left(z_{2}\right)$, obeys

$$
\begin{aligned}
\varepsilon_{f}\left(z_{2}\right)= & E_{f}-C_{1} C_{2} e R_{1} I_{0}-C_{1} C_{2} e R_{2} I_{0}+\left(S_{1} C_{1} C_{2}+S_{2} C_{2}+S_{f}\right) z_{2}+\left(-F_{1} F_{2} C_{1} C_{2} e Z_{1}-F_{3} C_{1} C_{2} e Z_{2}\right. \\
& \left.+i F_{1} F_{2} C_{1}^{3} C_{2}^{2} T_{2} e^{2} Z_{1} Z_{2} k_{0} I_{0}+i G_{3} C_{1} C_{2} / I_{0}+F_{1} G_{2} C_{1}^{3} C_{2}^{2} T_{2} e Z_{1} k_{0}\right) \Delta I e^{-i k_{0}\left(C_{1} C_{2} z_{2}+C_{1}^{2} C_{2} T_{2} e R_{1} I_{0}\right)} .
\end{aligned}
$$

For two-stage compression, the current and average-energy modulations given by Eqs. (B22), (B25), and (B28) obey

$$
\begin{gathered}
T_{I I} \equiv \frac{\Delta I / I_{\text {out }}}{\Delta I / I_{\text {in }}}=F_{3}-\frac{i F_{1} F_{2} C_{1}^{2} C_{2} e Z_{1} I_{0} k_{0} R_{56}^{(2)}}{E_{2}} \\
T_{E I} \equiv \frac{\Delta E / E_{\text {out }}}{\Delta I / I_{\text {in }}}=-\frac{C_{1} C_{2}\left(F_{1} F_{2} Z_{1}+F_{3} Z_{2}\right) e I_{0}}{E_{\text {out }}}+\frac{F_{1} G_{2} C_{1}^{3} C_{2}^{2} e Z_{1} I_{0} k_{0} R_{56}^{(2)}}{E_{2} E_{\text {out }}}+\frac{i F_{1} F_{2} C_{1}^{3} C_{2}^{2} e^{2} Z_{1} Z_{2} I_{0}^{2} k_{0} R_{56}^{(2)}}{E_{2} E_{\text {out }}}+\frac{i G_{3} C_{1} C_{2}}{E_{\text {out }}} .
\end{gathered}
$$

Equations (B29) and (B30) describe the modulation at any location after compression in $\mathrm{BC} 2$, provided that $E_{\text {out }}$ is the nominal energy at that location and $Z_{2}$ is the longitudinal impedance experienced after compression in $\mathrm{BC} 2$, prior to reaching that location.

\section{Energy modulation}

Consider an initial symmetric distribution of energies $f(\delta)$, where $\delta$ is the initial energy deviation from that of a cold, chirped beam with energy modulation. For constant current,

$$
I(z)=I_{0} .
$$

We again assume that the current distribution at the entrance of BC1 is $I(z, \delta)=I(z) f(\delta)$, where $I(z)$ is the bunch current. At the entrance of $\mathrm{BC} 1$, let $\varepsilon(z, \delta)$ equal the energy at coordinates $(z, \delta)$, while the average energy at longitudinal position $z$ is denoted $\varepsilon(z)$. For an energy modulation of $\Delta E e^{-i \omega_{0} t}=\Delta E e^{-i k_{0} z}$,

$$
\begin{gathered}
\varepsilon(z, \delta)=E_{1}+S_{1} z+\Delta E e^{-i k_{0} z}+\delta \\
\varepsilon(z)=\int \varepsilon(z, \delta) f(\delta) d \delta=E_{1}+S_{1} z+\Delta E e^{-i k_{0} z}
\end{gathered}
$$

The compression in $\mathrm{BC} 1$ gives the transformation

$$
\begin{aligned}
z_{1}(z, \delta) & =z+T_{1}\left[\varepsilon(z, \delta)-E_{1}\right] \\
& =z / C_{1}+T_{1} \delta+T_{1} \Delta E e^{-i k_{0} z},
\end{aligned}
$$

with derivative

$$
\partial z_{1} / \partial z=1 / C_{1}-i k_{0} T_{1} \Delta E e^{-i k_{0} z} .
$$

When the real part of this derivative is zero, upright bunch regions and current spikes occur after $\mathrm{BC} 1$. The following equations are valid to first order in $\Delta E$. The inverse of
Eq. (B34) is

$$
z\left(z_{1}, \delta\right)=C_{1} z_{1}-C_{1} T_{1} \delta-C_{1} T_{1} \Delta E e^{-i k_{0}\left(C_{1} z_{1}-C_{1} T_{1} \delta\right)} .
$$

After $\mathrm{BC} 1$, the current density in coordinates $\left(z_{1}, \delta\right)$ is

$$
\begin{aligned}
I_{1}\left(z_{1}, \delta\right) & =I\left[z\left(z_{1}, \delta\right), \delta\right] /\left(\partial z_{1} / \partial z\right) \\
& =C_{1} I_{0}\left(1+i C_{1} T_{1} k_{0} \Delta E e^{-i k_{0}\left(C_{1} z_{1}-C_{1} T_{1} \delta\right)}\right) f(\delta) .
\end{aligned}
$$

Therefore, the current is

$$
\begin{aligned}
I_{1}\left(z_{1}\right) & =\int I_{1}\left(z_{1}, \delta\right) d \delta \\
& =C_{1} I_{0}\left(1+i F_{1} C_{1} T_{1} k_{0} \Delta E e^{-i k_{0} C_{1} z_{1}}\right) .
\end{aligned}
$$

The bunch passes through the CSR and CER impedance upstream of the L1 linac, $\hat{Z}_{1}(k)$, where $\hat{Z}_{1}(0) \equiv \hat{R}_{1}$ and $\hat{Z}_{1}\left(C_{1} k_{0}\right) \equiv \hat{Z}_{1}$. The energy at the entrance of the L1 linac is

$$
\begin{aligned}
\varepsilon_{1}\left(z_{1}, \delta\right)= & \varepsilon\left[z\left(z_{1}, \delta\right), \delta\right]-(e / 2 \pi) \int d k \hat{Z}_{1}(k) I_{1}(k) e^{-i k z_{1}} \\
= & E_{1}+S_{1} C_{1} z_{1}+C_{1} \delta+C_{1} \Delta E e^{-i k_{0}\left(C_{1} z_{1}-C_{1} T_{1} \delta\right)} \\
& -e C_{1} I_{0}\left(\hat{R}_{1}+i F_{1} C_{1} T_{1} \hat{Z}_{1} k_{0} \Delta E e^{-i k_{0} C_{1} z_{1}}\right) .
\end{aligned}
$$

The average electron energy at coordinate $z_{1}, \varepsilon_{1}\left(z_{1}\right)$, obeys $\varepsilon_{1}\left(z_{1}\right) I_{1}\left(z_{1}\right)=\int d \delta \varepsilon_{1}\left(z_{1}, \delta\right) I_{1}\left(z_{1}, \delta\right)$, so that

$$
\begin{aligned}
\varepsilon_{1}\left(z_{1}\right)= & E_{1}+S_{1} C_{1} z_{1}+C_{1}\left(F_{1}-G_{1} C_{1} T_{1} k_{0}\right) \Delta E e^{-i k_{0} C_{1} z_{1}} \\
& -e C_{1} I_{0}\left(\hat{R}_{1}+i F_{1} C_{1} T_{1} \hat{Z}_{1} k_{0} \Delta E e^{-i k_{0} C_{1} z_{1}}\right) . \text { (B40) }
\end{aligned}
$$

After passing through the $\mathrm{L} 1$ linac between $\mathrm{BC} 1$ and $\mathrm{BC} 2$, the energy becomes 
$\varepsilon_{L}\left(z_{1}, \delta\right)=E_{2}+\left(S_{1} C_{1}+S_{2}\right) z_{1}+C_{1} \delta$

$$
\begin{aligned}
& +C_{1} \Delta E e^{-i k_{0}\left(C_{1} z_{1}-C_{1} T_{1} \delta\right)} \\
& -e C_{1} I_{0}\left(R_{1}+i F_{1} C_{1} T_{1} Z_{1} k_{0} \Delta E e^{-i k_{0} C_{1} z_{1}}\right) .
\end{aligned}
$$

The average electron energy at coordinate $z_{1}, \varepsilon_{L}\left(z_{1}\right)$, obeys

$$
\begin{aligned}
\varepsilon_{L}\left(z_{1}\right)= & E_{2}+\left(S_{1} C_{1}+S_{2}\right) z_{1} \\
& +C_{1}\left(F_{1}-G_{1} C_{1} T_{1} k_{0}\right) \Delta E e^{-i k_{0} C_{1} z_{1}} \\
& -e C_{1} I_{0}\left(R_{1}+i F_{1} C_{1} T_{1} Z_{1} k_{0} \Delta E e^{-i k_{0} C_{1} z_{1}}\right)
\end{aligned}
$$

For single-stage compression, the current and averageenergy modulations obey

$$
\begin{gathered}
T_{I E} \equiv \frac{\Delta I / I_{\text {out }}}{\Delta E / E_{\text {in }}}=i F_{1} C_{1} k_{0} R_{56}^{(1)}, \quad(\mathrm{B} 43) \\
T_{E E} \equiv \frac{\Delta E / E_{\text {out }}}{\Delta E / E_{\text {in }}} \\
=\frac{F_{1} C_{1} E_{1}}{E_{\text {out }}}-\frac{G_{1} C_{1}^{2} k_{0} R_{56}^{(1)}}{E_{\text {out }}}-\frac{i F_{1} C_{1}^{2} e Z_{1} I_{0} k_{0} R_{56}^{(1)}}{E_{\text {out }}} \\
z_{1}\left(z_{2}, \delta\right)=C_{2} z_{2}+C_{1} C_{2} T_{2} e R_{1} I_{0}-C_{1} C_{2} T_{2} \delta-C_{1} C_{2} T_{2} \Delta E e^{-i k_{0}\left[C_{1} C_{2} z_{2}+C_{1}^{2} C_{2} T_{2} e R_{1} I_{0}-\left(C_{1} T_{1}+C_{1}^{2} C_{2} T_{2}\right) \delta\right]} \\
+i F_{1} C_{1}^{2} C_{2} T_{1} T_{2} e Z_{1} I_{0} k_{0} \Delta E e^{-i k_{0}\left(C_{1} C_{2} z_{2}+C_{1}^{2} C_{2} T_{2} e R_{1} I_{0}-C_{1}^{2} C_{2} T_{2} \delta\right)}
\end{gathered}
$$

Equations (B43) and (B44) describe the modulation at any location between compression in $\mathrm{BC} 1$ and the entrance of BC2, provided that $E_{\text {out }}$ is the nominal energy at that location and $Z_{1}$ is the longitudinal impedance experienced after compression in $\mathrm{BC} 1$, prior to reaching that location. The compression in $\mathrm{BC} 2$ gives the transformation

$$
\begin{aligned}
z_{2}\left(z_{1}, \delta\right)= & z_{1}+T_{2}\left[\varepsilon_{L}\left(z_{1}, \delta\right)-E_{2}\right] \\
= & z_{1} / C_{2}-C_{1} T_{2} e R_{1} I_{0}+C_{1} T_{2} \delta \\
& +C_{1} T_{2} \Delta E e^{-i k_{0}\left(C_{1} z_{1}-C_{1} T_{1} \delta\right)} \\
& -i F_{1} C_{1}^{2} T_{1} T_{2} e Z_{1} I_{0} k_{0} \Delta E e^{-i k_{0} C_{1} z_{1}}
\end{aligned}
$$

with inverse

Using Eqs. (B34) and (B45) to relate $z_{2}$ and $z$ gives

$$
z_{2}(z, \delta)=z / C_{1} C_{2}-C_{1} T_{2} e R_{1} I_{0}+\left(T_{1} / C_{2}+T_{2} C_{1}\right)\left(\delta+\Delta E e^{-i k_{0} z}\right)-i F_{1} C_{1}^{2} T_{1} T_{2} e Z_{1} I_{0} k_{0} \Delta E e^{-i k_{0}\left(z+C_{1} T_{1} \delta\right)}
$$

The inverse of Eq. (B47) is

$$
\begin{aligned}
z\left(z_{2}, \delta\right)= & C_{1} C_{2} z_{2}+C_{1}^{2} C_{2} T_{2} e R_{1} I_{0}-\left(C_{1} T_{1}+C_{1}^{2} C_{2} T_{2}\right) \delta-\left(C_{1} T_{1}+C_{1}^{2} C_{2} T_{2}\right) \Delta E e^{-i k_{0}\left[C_{1} C_{2} z_{2}+C_{1}^{2} C_{2} T_{2} e R_{1} I_{0}-\left(C_{1} T_{1}+C_{1}^{2} C_{2} T_{2}\right) \delta\right]} \\
& +i F_{1} C_{1}^{3} C_{2} T_{1} T_{2} e Z_{1} I_{0} k_{0} \Delta E e^{-i k_{0}\left(C_{1} C_{2} z_{2}+C_{1}^{2} C_{2} T_{2} e R_{1} I_{0}-C_{1}^{2} C_{2} T_{2} \delta\right)}
\end{aligned}
$$

while its derivative is

$$
\partial z_{2} / \partial z(z, \delta)=\left(C_{1} C_{2}\right)^{-1}\left[1-i\left(C_{1} T_{1}+C_{1}^{2} C_{2} T_{2}\right) k_{0} \Delta E e^{-i k_{0} z}-F_{1} C_{1}^{3} C_{2} T_{1} T_{2} e Z_{1} I_{0} k_{0}^{2} \Delta E e^{-i k_{0}\left(z+C_{1} T_{1} \delta\right)}\right]
$$

Therefore, the current distribution is

$$
\begin{aligned}
I_{2}\left(z_{2}, \delta\right)= & I\left[z\left(z_{2}, \delta\right), \delta\right] /\left(\partial z_{2} / \partial z\right) \\
= & C_{1} C_{2} I_{0} f(\delta)\left[1+i\left(C_{1} T_{1}+C_{1}^{2} C_{2} T_{2}\right) k_{0} \Delta E e^{-i k_{0} z\left(z_{2}, \delta\right)}+F_{1} C_{1}^{3} C_{2} T_{1} T_{2} e Z_{1} I_{0} k_{0}^{2} \Delta E e^{-i k_{0}\left[z\left(z_{2}, \delta\right)+C_{1} T_{1} \delta\right]}\right] \\
= & C_{1} C_{2} I_{0} f(\delta)\left[1+i\left(C_{1} T_{1}+C_{1}^{2} C_{2} T_{2}\right) k_{0} \Delta E e^{-i k_{0}\left[C_{1} C_{2} z_{2}+C_{1}^{2} C_{2} T_{2} e R_{1} I_{0}-\left(C_{1} T_{1}+C_{1}^{2} C_{2} T_{2}\right) \delta\right]}\right. \\
& \left.+F_{1} C_{1}^{3} C_{2} T_{1} T_{2} e Z_{1} I_{0} k_{0}^{2} \Delta E e^{-i k_{0}\left(C_{1} C_{2} z_{2}+C_{1}^{2} C_{2} T_{2} e R_{1} I_{0}-C_{1}^{2} C_{2} T_{2} \delta\right)}\right] .
\end{aligned}
$$

This gives the current profile as 


$$
\begin{aligned}
I_{2}\left(z_{2}\right)= & \int I_{2}\left(z_{2}, \delta\right) d \delta \\
= & C_{1} C_{2} I_{0}\left[1+i\left(C_{1} T_{1}+C_{1}^{2} C_{2} T_{2}\right) F_{3} k_{0} \Delta E e^{-i k_{0}\left(C_{1} C_{2} z_{2}+C_{1}^{2} C_{2} T_{2} e R_{1} I_{0}\right)}\right. \\
& \left.+F_{1} F_{2} C_{1}^{3} C_{2} T_{1} T_{2} e Z_{1} I_{0} k_{0}^{2} \Delta E e^{-i k_{0}\left(C_{1} C_{2} z_{2}+C_{1}^{2} C_{2} T_{2} e R_{1} I_{0}\right)}\right] .
\end{aligned}
$$

After passing through the CSR and CER impedance upstream of the L2 linac, $\hat{Z}_{2}(k)$, where $\hat{Z}_{2}(0) \equiv \hat{R}_{2}$ and $\hat{Z}_{2}\left(C_{1} C_{2} k_{0}\right) \equiv$ $\hat{Z}_{2}$, the energy is

$$
\begin{aligned}
\varepsilon_{2}\left(z_{2}, \delta\right)= & \varepsilon_{L}\left[z_{1}\left(z_{2}, \delta\right), \delta\right]-(e / 2 \pi) \int d k \hat{Z}_{2}(k) I_{2}(k) e^{-i k z_{2}} \\
= & E_{2}-C_{1} C_{2} e R_{1} I_{0}-C_{1} C_{2} e \hat{R}_{2} I_{0}+\left(S_{1} C_{1}+S_{2}\right) C_{2} z_{2}+C_{1} C_{2} \delta \\
& +C_{1} C_{2} \Delta E e^{-i k_{0}\left[C_{1} C_{2} z_{2}+C_{1}^{2} C_{2} T_{2} e R_{1} I_{0}-\left(C_{1} T_{1}+C_{1}^{2} C_{2} T_{2}\right) \delta\right]}-i F_{1} C_{1}^{2} C_{2} T_{1} e Z_{1} I_{0} k_{0} \Delta E e^{-i k_{0}\left(C_{1} C_{2} z_{2}+C_{1}^{2} C_{2} T_{2} e R_{1} I_{0}-C_{1}^{2} C_{2} T_{2} \delta\right)} \\
& -i F_{3} C_{1} C_{2}\left(C_{1} T_{1}+C_{1}^{2} C_{2} T_{2}\right) e \hat{Z}_{2} I_{0} k_{0} \Delta E e^{-i k_{0}\left(C_{1} C_{2} z_{2}+C_{1}^{2} C_{2} T_{2} e R_{1} I_{0}\right)} \\
& -F_{1} F_{2} C_{1}^{4} C_{2}^{2} T_{1} T_{2} e^{2} Z_{1} \hat{Z}_{2} I_{0}^{2} k_{0}^{2} \Delta E e^{-i k_{0}\left(C_{1} C_{2} z_{2}+C_{1}^{2} C_{2} T_{2} e R_{1} I_{0}\right)}
\end{aligned}
$$

The average energy of electrons at coordinate $z_{2}, \varepsilon_{2}\left(z_{2}\right)$, obeys $\varepsilon_{2}\left(z_{2}\right) I_{2}\left(z_{2}\right)=\int d \delta \varepsilon_{2}\left(z_{2}, \delta\right) I_{2}\left(z_{2}, \delta\right)$. This gives

$$
\begin{aligned}
\varepsilon_{2}\left(z_{2}\right)= & E_{2}-C_{1} C_{2} e R_{1} I_{0}-C_{1} C_{2} e \hat{R}_{2} I_{0}+\left(S_{1} C_{1}+S_{2}\right) C_{2} z_{2}+\left[F_{3} C_{1} C_{2}-i F_{1} F_{2} C_{1}^{2} C_{2} T_{1} e Z_{1} I_{0} k_{0}\right. \\
& -i F_{3} C_{1} C_{2}\left(C_{1} T_{1}+C_{1}^{2} C_{2} T_{2}\right) e \hat{Z}_{2} I_{0} k_{0}-F_{1} F_{2} C_{1}^{4} C_{2}^{2} T_{1} T_{2} e^{2} Z_{1} \hat{Z}_{2} I_{0}^{2} k_{0}^{2}-G_{3} C_{1} C_{2}\left(C_{1} T_{1}+C_{1}^{2} C_{2} T_{2}\right) k_{0} \\
& \left.+i F_{1} G_{2} C_{1}^{4} C_{2}^{2} T_{1} T_{2} e Z_{1} I_{0} k_{0}^{2}\right] \Delta E e^{-i k_{0}\left(C_{1} C_{2} z_{2}+C_{1}^{2} C_{2} T_{2} e R_{1} I_{0}\right)} .
\end{aligned}
$$

After passing through the L2 linac, the final energy is

$$
\begin{aligned}
\varepsilon_{f}\left(z_{2}, \delta\right)= & E_{f}-C_{1} C_{2} e R_{1} I_{0}-C_{1} C_{2} e R_{2} I_{0}+\left(S_{1} C_{1} C_{2}+S_{2} C_{2}+S_{f}\right) z_{2}+C_{1} C_{2} \delta \\
& +C_{1} C_{2} \Delta E e^{-i k_{0}\left[C_{1} C_{2} z_{2}+C_{1}^{2} C_{2} T_{2} e R_{1} I_{0}-\left(C_{1} T_{1}+C_{1}^{2} C_{2} T_{2}\right) \delta\right]}-i F_{1} C_{1}^{2} C_{2} T_{1} e Z_{1} I_{0} k_{0} \Delta E e^{-i k_{0}\left(C_{1} C_{2} z_{2}+C_{1}^{2} C_{2} T_{2} e R_{1} I_{0}-C_{1}^{2} C_{2} T_{2} \delta\right)} \\
& -i F_{3} C_{1} C_{2}\left(C_{1} T_{1}+C_{1}^{2} C_{2} T_{2}\right) e Z_{2} I_{0} k_{0} \Delta E e^{-i k_{0}\left(C_{1} C_{2} z_{2}+C_{1}^{2} C_{2} T_{2} e R_{1} I_{0}\right)} \\
& -F_{1} F_{2} C_{1}^{4} C_{2}^{2} T_{1} T_{2} e^{2} Z_{1} Z_{2} I_{0}^{2} k_{0}^{2} \Delta E e^{-i k_{0}\left(C_{1} C_{2} z_{2}+C_{1}^{2} C_{2} T_{2} e R_{1} I_{0}\right)}
\end{aligned}
$$

The average energy at coordinate $z_{2}, \varepsilon_{f}\left(z_{2}\right)$, obeys

$$
\begin{aligned}
\varepsilon_{f}\left(z_{2}\right)= & E_{f}-C_{1} C_{2} e R_{1} I_{0}-C_{1} C_{2} e R_{2} I_{0}+\left(S_{1} C_{1} C_{2}+S_{2} C_{2}+S_{f}\right) z_{2}+\left[F_{3} C_{1} C_{2}-i F_{1} F_{2} C_{1}^{2} C_{2} T_{1} e Z_{1} I_{0} k_{0}\right. \\
& -i F_{3} C_{1} C_{2}\left(C_{1} T_{1}+C_{1}^{2} C_{2} T_{2}\right) e Z_{2} I_{0} k_{0}-F_{1} F_{2} C_{1}^{4} C_{2}^{2} T_{1} T_{2} e^{2} Z_{1} Z_{2} I_{0}^{2} k_{0}^{2}-G_{3} C_{1} C_{2}\left(C_{1} T_{1}+C_{1}^{2} C_{2} T_{2}\right) k_{0} \\
& \left.+i F_{1} G_{2} C_{1}^{4} C_{2}^{2} T_{1} T_{2} e Z_{1} I_{0} k_{0}^{2}\right] \Delta E e^{-i k_{0}\left(C_{1} C_{2} z_{2}+C_{1}^{2} C_{2} T_{2} e R_{1} I_{0}\right)}
\end{aligned}
$$

For two-stage compression, the current and average-energy modulations given by Eqs. (B51), (B53), and (B55) obey

$$
\begin{gathered}
T_{I E} \equiv \frac{\Delta I / I_{\text {out }}}{\Delta E / E_{\text {in }}}=\frac{F_{1} F_{2} C_{1}^{3} C_{2} e Z_{1} I_{0} k_{0}^{2} R_{56}^{(1)} R_{56}^{(2)}}{E_{2}}+i F_{3} C_{1} k_{0} R_{56}^{(1)}+\frac{i F_{3} C_{1}^{2} C_{2} k_{0} R_{56}^{(2)} E_{1}}{E_{2}} \\
T_{E E} \equiv \frac{\Delta E / E_{\text {out }}}{\Delta E / E_{\text {in }}}=\frac{F_{3} C_{1} C_{2} E_{1}}{E_{\text {out }}}-\frac{F_{1} F_{2} C_{1}^{4} C_{2}^{2} e^{2} Z_{1} Z_{2} I_{0}^{2} k_{0}^{2} R_{56}^{(1)} R_{56}^{(2)}}{E_{2} E_{\text {out }}}-\frac{G_{3} C_{1}^{2} C_{2} k_{0} R_{56}^{(1)}}{E_{\text {out }}}-\frac{G_{3} C_{1}^{3} C_{2}^{2} k_{0} R_{56}^{(2)} E_{1}}{E_{2} E_{\text {out }}} \\
-\frac{i F_{1} F_{2} C_{1}^{2} C_{2} e Z_{1} I_{0} k_{0} R_{56}^{(1)}}{E_{\text {out }}}-\frac{i F_{3} C_{1}^{2} C_{2} e Z_{2} I_{0} k_{0} R_{56}^{(1)}}{E_{\text {out }}}-\frac{i F_{3} C_{1}^{3} C_{2}^{2} e Z_{2} I_{0} k_{0} R_{56}^{(2)} E_{1}}{E_{2} E_{\text {out }}}+\frac{i F_{1} G_{2} C_{1}^{4} C_{2}^{2} e Z_{1} I_{0} k_{0}^{2} R_{56}^{(1)} R_{56}^{(2)}}{E_{2} E_{\text {out }}}
\end{gathered}
$$

Equations (B56) and (B57) describe the modulation at any location after compression in BC2, provided that $E_{\text {out }}$ is the nominal energy at that location and $Z_{2}$ is the longitudinal impedance experienced after compression in $\mathrm{BC} 2$, prior to reaching that location. 
[1] J. Arthur et al., SLAC Report No. SLAC-R-593, 2002.

[2] M. Borland, Y.-C. Chae, S. Milton, R. Soliday, V. Bharadwaj, P. Emma, P. Krejcik, C. Limborg, H.-D. Nuhn, and M. Woodley, in Proceedings of the 2001 Particle Accelerator Conference, Chicago (IEEE, Piscataway, NJ, 2001), p. 2707.

[3] M. Borland, Y. C. Chae, P. Emma, J. W. Lewellen, V. Bharadwaj, W. M. Fawley, P. Krejcik, C. Limborg, S. V. Milton, H.-D. Nuhn, R. Soliday, and M. Woodley, Nucl. Instrum. Methods Phys. Res., Sect. A 483, 268 (2002).

[4] E. L. Saldin, E. A. Schneidmiller, and M. V. Yurkov, Nucl. Instrum. Methods Phys. Res., Sect. A 490, 1 (2002).

[5] S. Heifets, G. Stupakov, and S. Krinsky, Phys. Rev. ST Accel. Beams 5, 064401 (2002).

[6] Z. Huang and K.-J. Kim, Phys. Rev. ST Accel. Beams 5, 074401 (2002).

[7] J. Wu, P. Emma, Z. Huang, C. Limborg, and M. Borland, in Proceedings of the XXII International Linear Accelerator Conference (LINAC 2004), Lubeck, Germany (DESY, Hamburg, 2004), p. 390.

[8] Z. Huang, M. Borland, P. Emma, J. Wu, C. Limborg, G. Stupakov, and J. Welch, Phys. Rev. ST Accel. Beams 7, 074401 (2004).

[9] E. L. Saldin, E. A. Schneidmiller, and M. V. Yurkov, Nucl. Instrum. Methods Phys. Res., Sect. A 528, 355 (2004).

[10] M. Venturini, R. Warnock, and A. Zholents, Phys. Rev. ST Accel. Beams 10, 054403 (2007).

[11] J. B. Murphy, S. Krinsky, and R. L. Gluckstern, Part. Accel. 57, 9 (1997).

[12] O. V. Chubar and N. V. Smolyakov, J. Opt. 24, 117 (1993).

[13] R. A. Bosch and O. V. Chubar, in Synchrotron Radiation Instrumentation: Tenth US National Conference, edited by E. Fontes, AIP Conf. Proc. No. 417 (AIP, New York, 1997), p. 35.

[14] R. A. Bosch, Phys. Rev. ST Accel. Beams 10, 050701 (2007).

[15] J. B. Rosenzweig, M. Dunning, E. Hemsing, G. Andonian, A. M. Cook, A. Murokh, S. Reiche, D. Schiller, M. Babzien, K. Kusche, V. Yakimenko, L. Palumbo, and C. Vicario, in Advanced Accelerator Concepts, edited by M. Conde and C. Eyberger, AIP Conf. Proc. No. 877 (AIP, New York, 2006), p. 642.

[16] J. J. Bisognano, R. A. Bosch, M. A. Green, K. D. Jacobs, K. J. Kleman, R. A. Legg, J. Chen, W. S. Graves, F. X. Kärtner, and J. Kim, in Proceedings of the 2007 Particle Accelerator Conference, Albuquerque, NM (IEEE, Piscataway, NJ, 2007), p. 1281.
[17] M. Borland, Advanced Photon Source Light Source Note LS-287, 2000.

[18] H. Grote, E. Keil, T. O. Raubenheimer, and M. Woodley, in Proceedings of the 7th European Particle Accelerator Conference, Vienna (EPS, Geneva, 2000), p. 1390.

[19] J. Rosenzweig and L. Serafini, Phys. Rev. E 49, 1599 (1994).

[20] R. L. Gluckstern, Phys. Rev. D 39, 2780 (1989).

[21] K. Yokoya and K. L.F. Bane, in Proceedings of the 1999 Particle Accelerator Conference, New York (IEEE, Piscataway, NJ, 1999), p. 1725.

[22] D. Proch, DESY Report No. TESLA-Report 1994-13, 1994.

[23] A. Novokhatski and A. Mosnier, in Proceedings of the 1997 Particle Accelerator Conference, Vancouver, Canada (IEEE, Piscataway, NJ, 1998), p. 467.

[24] D. J. Wingham, Phys. Rev. D 35, 2584 (1987).

[25] M. Dohlus and T. Limberg, Nucl. Instrum. Methods Phys. Res., Sect. A 393, 494 (1997).

[26] E. L. Saldin, E. A. Schneidmiller, and M. V. Yurkov, Nucl. Instrum. Methods Phys. Res., Sect. A 398, 373 (1997).

[27] G. Stupakov and P. Emma, in Proceedings of the Eighth European Particle Accelerator Conference, Paris (EPSIGA/CERN, Geneva, 2002), p. 1479.

[28] D. R. Douglas, J. Kewisch, and R. C. York, in Proceedings of the 1988 Linear Accelerator Conference, Williamsburg, VA, 1988 (CEBAF Report No. 89-001, 1988), p. 328.

[29] J. H. Halton, Numer. Math. 2, 84 (1960).

[30] K. L.F. Bane and P. Emma, in Proceedings of the 2005 Particle Accelerator Conference, Knoxville, TN (IEEE, Piscataway, NJ, 2005), p. 4266.

[31] M. Cornacchia, S. Di Mitri, G. Penco, and A. A. Zholents, Phys. Rev. ST Accel. Beams 9, 120701 (2006).

[32] H. Schlarb, Ch. Gerth, W. Koprek, F. Loehl, and E. Vogel, in Proceedings of the 8th European Workshop on Beam Diagnostics and Instrumentation for Particle Accelerators, Venice (Sincrotrone Trieste, Venice, 2007).

[33] I.S. Gradshteyn and I. M. Ryzhik, Table of Integrals, Series, and Products (Academic Press, San Diego, 1994), 5th ed., Eq. 3.896 .4 on p. 515 and Eq. 3.952.1 on p. 529.

[34] I.S. Gradshteyn and I. M. Ryzhik, Table of Integrals, Series, and Products (Ref. [33]), Eqs. 2.261 and 2.262.3 on p. 99.

[35] I. S. Gradshteyn and I. M. Ryzhik, Table of Integrals, Series, and Products (Ref. [33]), Eq. 3.752 .2 on p. 456 and Eq. 3.771 .10 on p. 465. 